\title{
Regularity estimates in weighted Morrey spaces for quasilinear elliptic equations
}

\author{
Giuseppe Di Fazio* $\quad$ Truyen Nguyen ${ }^{\dagger}$
}

\begin{abstract}
We study regularity for solutions of quasilinear elliptic equations of the form $\operatorname{div} \mathbf{A}(x, u, \nabla u)=\operatorname{div} \mathbf{F}$ in bounded domains in $\mathbb{R}^{n}$. The vector field $\mathbf{A}$ is assumed to be continuous in $u$, and its growth in $\nabla u$ is like that of the $p$-Laplace operator. We establish interior gradient estimates in weighted Morrey spaces for weak solutions $u$ to the equation under a small BMO condition in $x$ for A. As a consequence, we obtain that $\nabla u$ is in the classical Morrey space $\mathcal{M}^{q, \lambda}$ or weighted space $L_{w}^{q}$ whenever $|\mathbf{F}|^{\frac{1}{p-1}}$ is respectively in $\mathcal{M}^{q, \lambda}$ or $L_{w}^{q}$, where $q$ is any number greater than $p$ and $w$ is any weight in the Muckenhoupt class $A_{\frac{q}{p}}$. In addition, our two-weight estimate allows the possibility to acquire the regularity for $\nabla u$ in a weighted Morrey space that is different from the functional space that the data $|\mathbf{F}|^{\frac{1}{p-1}}$ belongs to.
\end{abstract}

2010 Mathematics Subject Classification. 30H35, 35B45, 35B65, 35J92.

Key words and phrases. elliptic equations, p-Laplacian, gradient estimates, Calderón-Zygmund estimates, weighted Morrey spaces, regularity, two-weight inequality, Muckenhoupt class.

\section{Introduction}

We investigate interior gradient estimates in weighted Morrey space for bounded weak solutions to the following general elliptic equations of $p$-Laplacian type

$$
\operatorname{div} \mathbf{A}(x, u, \nabla u)=\operatorname{div} \mathbf{F} \quad \text { in } \Omega
$$

when $\Omega$ is a bounded domain in $\mathbb{R}^{n}(n \geq 1)$ and the vector field $\mathbf{A}$ is only continuous in the $u$ variable and possibly discontinuous in the $x$ variable. Without loss of generality we take $\Omega$ to be the Euclidean ball $B_{10}:=\left\{x \in \mathbb{R}^{n}:|x|<10\right\}$, whose special radius is simply to avoid working with many fractions in our arguments later on. Let $\mathbb{K} \subset \mathbb{R}$ be an open interval and consider the general vector field

$$
\mathbf{A}=\mathbf{A}(x, z, \xi): B_{10} \times \overline{\mathbb{K}} \times \mathbb{R}^{n} \longrightarrow \mathbb{R}^{n}
$$

which is a Carathéodory map, that is, $\mathbf{A}(x, z, \xi)$ is measurable in $x$ for every $(z, \xi) \in \overline{\mathbb{K}} \times \mathbb{R}^{n}$ and continuous in $(z, \xi)$ for a.e. $x$. We assume that $\xi \mapsto \mathbf{A}(x, z, \xi)$ is differentiable on $\mathbb{R}^{n} \backslash\{0\}$ for a.e. $x$ and all $z \in \overline{\mathbb{K}}$. Also, there exist constants $\Lambda>0,1<p<\infty$, and a nondecreasing and right continuous function $\omega:[0, \infty) \rightarrow[0, \infty)$ with $\omega(0)=0$ such that the following conditions are satisfied for a.e. $x \in B_{10}$ and all $z \in \overline{\mathbb{K}}$ :

$$
\begin{array}{ll}
\left\langle\partial_{\xi} \mathbf{A}(x, z, \xi) \eta, \eta\right\rangle \geq \Lambda^{-1}|\xi|^{p-2}|\eta|^{2} & \forall \xi \in \mathbb{R}^{n} \backslash\{0\} \text { and } \forall \eta \in \mathbb{R}^{n}, \\
|\mathbf{A}(x, z, \xi)|+|\xi|\left|\partial_{\xi} \mathbf{A}(x, z, \xi)\right| \leq \Lambda|\xi|^{p-1} & \forall \xi \in \mathbb{R}^{n}, \\
\left|\mathbf{A}\left(x, z_{1}, \xi\right)-\mathbf{A}\left(x, z_{2}, \xi\right)\right| \leq \Lambda|\xi|^{p-1} \omega\left(\left|z_{1}-z_{2}\right|\right) & \forall z_{1}, z_{2} \in \overline{\mathbb{K}} \text { and } \forall \xi \in \mathbb{R}^{n} .
\end{array}
$$

\footnotetext{
${ }^{\dagger}$ The research of the author is supported in part by a grant from the Simons Foundation (\# 318995) and by the UA Faculty Research grant FRG (\# 207359).
} 
The class of equations of the form (1.1) with A satisfying (1.2)-1.4 contains the well known $p$-Laplace equations and, more generally, equations of the type

$$
\operatorname{div} \mathbf{A}(x, \nabla u)=\operatorname{div} \mathbf{F} \quad \text { in } \Omega .
$$

Gradient estimates for (1.5) with discontinuous coefficient have been studied extensively by several authors [3, 4, 7, -10, 13, 18, 20, 22, 24, 25, 33, 36]. Some of these developments rely essentially on the perturbation method developed by Caffarelli and Peral [4], which allows ones to deal with discontinuous coefficient and highly nonlinear structure in gradient of equation (1.5).

In this paper we study general quasilinear equation (1.1) when the principal part also depends on the $z$ variable. In the case $\mathbf{A}$ is Lipschitz continuous in both $x$ and $z$ variables, the interior $C^{1, \alpha}$ regularity for locally bounded weak solutions to the corresponding homogeneous equation was established by DiBenedetto [6] and Tolksdorf [35] extending the celebrated $C^{1, \alpha}$ estimates by Uraltceva [37], Uhlenbeck [36], Evans [11], and Lewis [23] for the homogeneous $p$-Laplace equation. When $\mathbf{A}$ is not necessarily continuous in $x$ but has sufficiently small BMO oscillation, it was established in [29] that: $|\mathbf{F}|^{\frac{1}{p-1}} \in L_{l o c}^{q} \Longrightarrow \nabla u \in L_{l o c}^{q}$ for any $q>p$. It is required in [29] that $\mathbf{A}$ is Lipschitz continuous in the $z$ variable since the uniqueness property for the frozen equation is used there. However, this condition is weaken in a recent paper [2] allowing only Hölder continuity in $z$ for $\mathbf{A}$ (i.e. $\omega(s)=s^{\alpha}$ for some $\alpha \in(0,1)$ in $(1.4)$ ).

Our purpose of the current work is to extend the mentioned result in [2,29] by deriving interior estimates in weighted Morrey spaces for gradients of locally bounded weak solutions to nonhomogeneous equation (1.1). In order to state our main results, let us recall the so-called Muckenhoupt class of $A_{s}$ weights. By definition, a weight is a nonnegative locally integrable function on $\mathbb{R}^{n}$ that is positive almost everywhere. A weight $w$ belongs to the class $A_{s}, 1<s<\infty$, if

$$
[w]_{A_{s}}:=\sup \left(f_{B} w(x) d x\right)\left(f_{B} w(x)^{\frac{-1}{s-1}} d x\right)^{s-1}<\infty,
$$

where the supremum is taken over all balls $B$ in $\mathbb{R}^{n}$. We also say that $w$ belongs to the class $A_{\infty}$ if

$$
[w]_{A_{\infty}}:=\sup \left(f_{B} w(x) d x\right) \exp \left(f_{B} \log w(x)^{-1} d x\right)<\infty .
$$

Now let $U \subset \mathbb{R}^{n}$ be a bounded open set, $w$ be a weight, $1 \leq q<\infty$, and $\varphi$ be a positive function on the set of nonempty open balls in $\mathbb{R}^{n}$. A function $g: U \rightarrow \mathbb{R}$ is said to belong to the weighted space $L_{w}^{q}(U)$ if

$$
\|g\|_{L_{w}^{q}(U)}:=\left(\int_{U}|g(x)|^{q} w(x) d x\right)^{\frac{1}{q}}<\infty .
$$

We define the weighted Morrey space $\mathcal{M}_{w}^{q, \varphi}(U)$ to be the set of all functions $g \in L_{w}^{q}(U)$ satisfying

$$
\|g\|_{\mathcal{M}_{w}^{q, \varphi}(U)}:=\sup _{\bar{x} \in U, 0<r \leq \operatorname{diam}(U)}\left(\frac{\varphi\left(B_{r}(\bar{x})\right)}{w\left(B_{r}(\bar{x})\right)} \int_{B_{r}(\bar{x}) \cap U}|g(x)|^{q} w d x\right)^{\frac{1}{q}}<\infty .
$$

Notice that $\mathcal{M}_{w}^{q, \varphi}(U)=L_{w}^{q}(U)$ if $\varphi(B)=w(B)$, and we obtain the classical Morrey space $\mathcal{M}^{q, \lambda}(U)(0 \leq$ $\lambda \leq n)$ by taking $w=1$ and $\varphi(B)=|B|^{\frac{\lambda}{n}}$. It is worth observing that $\mathcal{M}^{q, \lambda}(U)=L^{q}(U)$ when $\lambda=n$ and $\mathcal{M}^{q, \lambda}(U)=L^{\infty}(U)$ when $\lambda=0$. For brevity, the Morrey space $\mathcal{M}_{w}^{q, \varphi}(U)$ with $w=1$ will be denoted by $\mathcal{M}^{q, \varphi}(U)$. Let us next introduce some slight restrictions on the function $\varphi$. 
Definition 1.1. Let $\varphi$ be a positive function on the set of nonempty open balls in $\mathbb{R}^{n}$. We say that $\varphi$ belongs to the class $\mathcal{B}_{\alpha}$ with $\alpha \geq 0$ if there exists $C>0$ such that

$$
\frac{\varphi\left(B_{r}(x)\right)}{\varphi\left(B_{s}(x)\right)} \leq C\left(\frac{r}{s}\right)^{\alpha}
$$

for every $x \in \mathbb{R}^{n}$ and every $0<r \leq s$. We define $\mathcal{B}_{+}:=\cup_{\alpha>0} \mathcal{B}_{\alpha} \subset \mathcal{B}_{0}$.

The function $\varphi(B):=|B|^{\frac{\alpha}{n}}$ with $\alpha>0$ is an example of functions in $\mathcal{B}_{+}$. Also for any weight $w \in A_{\infty}$, the function $\varphi(B):=w(B)$ belongs to the class $\mathcal{B}_{+}$due to the characterization (2.2) below. However, a member of $\mathcal{B}_{+}$does not need to be even a measure. Hereafter, for a ball $B \subset \mathbb{R}^{n}$ we set $\overline{\mathbf{A}}_{B}(z, \xi):=f_{B} \mathbf{A}(x, z, \xi) d x$. Also, the conjugate exponent of a number $l \in(1, \infty)$ is denoted by $l^{\prime}$. Our first main result is:

Theorem 1.2. Let A satisfy (1.2)-1.4 with $p>1$, and let $w$ be an $A_{s}$ weight for some $1<s<\infty$. Then for any $q \geq p, M_{0}>0$, and $\varphi \in \mathcal{B}_{+}$with $\sup _{x \in B_{1}} \varphi\left(B_{2}(x)\right)<\infty$, there exists a constant $\delta=$ $\delta\left(p, q, n, \omega, \Lambda, M_{0}, s,[w]_{A_{s}}\right)>0$ such that: if

$$
\sup _{0<\rho \leq 1} \sup _{y \in B_{\frac{15}{2}}} \sup _{z \in \mathbb{K} \cap\left[-M_{0}, M_{0}\right]} f_{B_{\rho}(y)}\left[\sup _{\xi \neq 0} \frac{\left|\mathbf{A}(x, z, \xi)-\overline{\mathbf{A}}_{B_{\rho}(y)}(z, \xi)\right|}{|\xi|^{p-1}}\right] d x \leq \delta,
$$

and $u$ is a weak solution of

$$
\operatorname{div} \mathbf{A}(x, u, \nabla u)=\operatorname{div} \mathbf{F} \quad \text { in } \quad B_{10}
$$

satisfying $\|u\|_{L^{\infty}\left(B_{10}\right)} \leq M_{0}$, we have

$$
\|\nabla u\|_{\mathcal{M}_{w}^{q, \varphi}\left(B_{1}\right)} \leq C\left(\|\nabla u\|_{L^{p}\left(B_{10}\right)}+\left\|\mathbf{M}_{B_{10}}\left(|\mathbf{F}|^{\frac{p}{p-1}}\right)\right\|_{\mathcal{M}^{1, \varphi^{q}}\left(B_{10}\right)}^{\frac{1}{p}}+\left\|\mathbf{M}_{B_{10}}\left(|\mathbf{F}|^{\frac{p}{p-1}}\right)\right\|_{\mathcal{M}_{w}^{\frac{q}{p}, \varphi}\left(B_{10}\right)}^{\frac{1}{p}}\right) .
$$

Here $\mathrm{M}_{B_{10}}$ denotes the centered Hardy-Littlewood maximal operator (see Definition 3.1), and $C>0$ is a constant depending only on $q, p, n, \omega, \Lambda, M_{0}, \varphi, s$, and $[w]_{A_{s}}$.

The above theorem holds true for any weight $w$ in the class $A_{\infty}$. When $q>p$ and certain additional information about the weights and $\varphi, \phi$ is given, we can further estimate the two quantities in (1.9) involving the maximal function of $|\mathbf{F}|^{\frac{p}{p-1}}$ to obtain:

Theorem 1.3 (weighted Morrey space estimate). Let $\mathbf{A}$ satisfy (1.2)-(1.4) with $p>1$. Let $q>p, w \in A_{\infty}$, $v \in A_{\frac{q}{p}}, \varphi \in \mathcal{B}_{+}$with $\sup _{x \in B_{1}} \varphi\left(B_{2}(x)\right)<\infty$, and $\phi \in \mathcal{B}_{0}$ satisfy

$$
\begin{aligned}
& {\left[w, v^{1-\left(\frac{q}{p}\right)^{\prime}}\right]_{\frac{q}{p}}:=\sup _{B}\left(f_{B} w d x\right)\left(f_{B} v^{1-\left(\frac{q}{p}\right)^{\prime}} d x\right)^{\frac{q}{p}-1}<\infty,} \\
& \frac{v(2 B)}{w(2 B)} \frac{1}{\phi(2 B)} \leq C_{*} \frac{1}{\varphi(B)} \text { for all balls } B \subset \mathbb{R}^{n} .
\end{aligned}
$$

Then for any $M_{0}>0$, there exists a small constant $\delta=\delta\left(p, q, n, \omega, \Lambda, M_{0},[w]_{A_{\infty}}\right)>0$ such that: if (1.7) holds and $u$ is a weak solution of (1.8) satisfying $\|u\|_{L^{\infty}\left(B_{10}\right)} \leq M_{0}$, we have

$$
\|\nabla u\|_{\mathcal{M}_{w}^{q, \varphi}\left(B_{1}\right)} \leq C\left(\|\nabla u\|_{L^{p}\left(B_{10}\right)}+\left\||\mathbf{F}|^{\frac{1}{p-1}}\right\|_{\mathcal{M}_{v}^{q, \phi}\left(B_{10}\right)}\right) .
$$

Here $C>0$ is a constant depending only on $q, p, n, \omega, \Lambda, M_{0}, \varphi, \phi, C_{*},[w]_{A_{\infty}},[v]_{A_{\frac{q}{p}}}$, and $\left[w, v^{1-\left(\frac{q}{p}\right)^{\prime}}\right]_{A_{\frac{q}{p}}}$. 
Remark 1.4. If $\phi(2 B) \leq C \varphi(B)$ for all balls $B$, then conditions 1.10)-1.11 imply that $v(x) \leq C C_{*} w(x)$ a.e. and $v \in A_{\frac{q}{p}}$ with $[v]_{A_{\frac{q}{p}}} \leq C C_{*}\left[w, v^{1-\left(\frac{q}{p}\right)^{\prime}}\right]_{A_{\frac{q}{p}}}$.

Estimate (1.12) is a two-weight inequality which allows the possibility to acquire the regularity for $\nabla u$ in a weighted Morrey space that is different from the functional space that the data $|\mathbf{F}|^{\frac{1}{p-1}}$ belongs to. In particular, the result in Theorem 1.3 is even new when applied to equations of special form (1.5) whose gradient estimates in the classical Morrey spaces are obtained in [24, 25, 33]. Our results also improve the $L^{q}$ gradient estimates established in [2, 29] for equation (1.1) since the principal part $\mathbf{A}(x, z, \xi)$ is merely assumed to be continuous in the $z$ variable instead of being Hölder continuous.

One of the main difficulties in proving the above main results is that equations of the form (1.8) are not invariant with respect to dilations and rescaling of domains due to the dependence of $\mathbf{A}(x, z, \xi)$ on the $z$ variable. To handle this issue, we use the key idea introduced in [17, 29] by enlarging the class of equations under consideration in a suitable way. Precisely, we consider the associated quasilinear elliptic equations with two parameters, i.e. equation (2.4) below. The class of these equations is the smallest one that is invariant with respect to dilations and rescaling of domains and that contains equations of the form (1.8). Given the invariant structure, we employ the direct argument in [1,2] to show that the gradient of the solution $u$ can be approximated by a bounded gradient in $L^{p}$ norm (see Proposition 4.1). It is essential for us that this approximation is uniform with respect to the two parameters. With this and through a standard procedure, we derive some density estimates and obtain Theorem 2.4 about gradient estimates in weighted $L^{q}$ spaces (see Subsections 5.1 and 5.2. This estimate plays a central role in proving our main results. Indeed, by using the trick in [25] we show in Subsection 5.3 that Theorem 1.2 can be derived as a consequence of Theorem 2.4. In order to prove Theorem 1.3, we need to further estimate the last two terms in inequality (1.9) involving the maximal function of $|\mathbf{F}|^{p^{\prime}}$. This is another difficulty that needs to overcome and it can be solved if one has a suitable estimate for the maximal function in weighted Morrey spaces. This type of estimate is proved by Chiarenza and Frasca [5] in the unweighted setting, and there are some recent works [15, 16, 21, 27] establishing the estimate in the weighted setting under certain conditions. However, they are inadequate for our purpose and we need to extend these results by attaining in Theorem 3.4 a weighted Morrey space estimate for the maximal function. This two-weight inequality holds true for quite general weights and we believe that it is of independent interest.

We end the introduction by pointing out that our method of establishing interior gradient estimates in weighted Morrey spaces could be combined with the boundary techniques used in [2, 3, 24, 25] to derive global estimates for Reifenberg flat domains and homogeneous Dirichlet boundary condition. Furthermore, by following [32] it might be possible to weaken the assumption $u \in L^{\infty}$ in Theorem 1.3 by assuming only that $u \in B M O$.

The organization of the paper is as follows. We recall some basic properties of $A_{s}$ weights in Subsection 2.1 and state a key result (Theorem 2.4) in Subsection 2.2 about gradient estimates in weighted $L^{q}$ spaces. In Section 3, we derive a weighted Morrey space estimate for the maximal function (see Theorem 3.4 and its corollaries). Section 4 is devoted to proving Proposition 4.1 which shows that gradients of weak solutions to two-parameter equation (2.4) can be approximated by bounded gradients under some smallness conditions. Using this crucial result, we establish in Section 5 some density estimates for gradients and then prove Theorem 2.4. In this same section, the main results stated in Theorem 1.2 and Theorem 1.3 are derived as consequences of Theorem 2.4 . 


\section{Preliminaries and a key result}

\subsection{Some basic properties of $A_{s}$ weights}

Let us recall some properties of weights which can be found in [14, Chapter 9]. Given a weight $w$ and a measurable set $E \subset \mathbb{R}^{n}$, we use the notation $d w(x)=w(x) d x$ and $w(E)=\int_{E} w(x) d x$.

Lemma 2.1. Let $w \in A_{s}$ for some $1<s<\infty$. Then

1) There exist $0<\beta \leq 1$ and $K>0$ depending only on $n$ and $[w]_{A_{s}}$ such that

$$
[w]_{A_{s}}^{-1}\left(\frac{|E|}{|B|}\right)^{s} \leq \frac{w(E)}{w(B)} \leq K\left(\frac{|E|}{|B|}\right)^{\beta}
$$

for all balls $B$ and all measurable sets $E \subset B$. In particular, $w$ is doubling with $w(2 B) \leq 2^{n s}[w]_{A_{s}} w(B)$.

2) The function $w^{1-s^{\prime}}$ is in $A_{s^{\prime}}$ with characteristic constant $\left[w^{1-s^{\prime}}\right]_{A_{s^{\prime}}}=[w]_{A_{s}}^{\frac{1}{s-1}}$.

3) The classes $A_{s}$ are increasing as s increases; precisely, for $1<s<q \leq \infty$ we have $[w]_{A_{q}} \leq[w]_{A_{s}}$.

4) For any $1<q \leq \infty$, we have $A_{q}=\bigcup_{1<s<q} A_{s}$.

Lemma 2.2 (Characterizations of $A_{\infty}$ weights). Suppose that $w$ is a weight. Then $w$ is in $A_{\infty}$ if and only if there exist $0<A, v<\infty$ such that for all balls $B$ and all measurable sets $E \subset B$ we have

$$
\frac{w(E)}{w(B)} \leq A\left(\frac{|E|}{|B|}\right)^{v}
$$

When $w \in A_{\infty}$, the above constants $A$ and $v$ depend only on $n$ and $[w]_{A_{\infty}}$. Conversely, given constants $A$ and $v$ satisfying $\left[2.2\right.$, we have $[w]_{A_{\infty}} \leq C(n, A, v)$.

\subsection{Quasilinear equations with two parameters}

Our goal is to derive interior gradient estimates for weak solutions to (1.8). Let us consider a function $u \in W_{l o c}^{1, p}\left(B_{r R}\right)$ such that $u(y) \in \overline{\mathbb{K}}$ for a.e. $y \in B_{r R}$ and $u$ satisfies $\operatorname{div} \mathbf{A}(y, u, \nabla u)=\operatorname{div} \mathbf{F}$ in $B_{r R}$ in the sense of distribution. Then the rescaled function

$$
v(x):=\frac{u(r x)}{\mu r} \quad \text { for } \quad r, \mu>0
$$

has the properties: $v(x) \in \frac{1}{\mu r} \overline{\mathbb{K}}$ for a.e. $x \in B_{R}$ and $v$ solves the equation

$$
\operatorname{div} \mathbf{A}_{\mu, r}(x, \mu r v, \nabla v)=\operatorname{div} \mathbf{F}_{\mu, r} \quad \text { in } \quad B_{R}
$$

in the distributional sense. Here,

$$
\mathbf{A}_{\mu, r}(x, z, \xi):=\frac{\mathbf{A}(r x, z, \mu \xi)}{\mu^{p-1}} \quad \text { and } \quad \mathbf{F}_{\mu, r}(x):=\frac{\mathbf{F}(r x)}{\mu^{p-1}} .
$$


It is clear that if $\mathbf{A}: B_{r R} \times \overline{\mathbb{K}} \times \mathbb{R}^{n} \longrightarrow \mathbb{R}^{n}$ satisfies conditions (1.2)-(1.4), then the rescaled vector field $\mathbf{A}_{\mu, r}: B_{R} \times \overline{\mathbb{K}} \times \mathbb{R}^{n} \longrightarrow \mathbb{R}^{n}$ also satisfies these conditions with the same constants.

The above observation shows that equations of type (1.8) are not invariant with respect to the standard scalings (2.3). This presents a serious obstacle in obtaining weighted $L^{q}$ estimates for their solutions by using the methods in [3, 24, 25]. Here we follow the idea in [17, 29] by considering associated quasilinear equations with two parameters

$$
\operatorname{div}\left[\frac{\mathbf{A}(x, \lambda \theta u, \lambda \nabla u)}{\lambda^{p-1}}\right]=\operatorname{div} \mathbf{F} \quad \text { in } B_{10}
$$

with $\lambda, \theta>0$. The class of these equations is the smallest one that is invariant with respect to the transformations (2.3) and that contains equations of type (1.8). Indeed, if $u$ solves (2.4) and $v$ is given by (2.3), then $v$ satisfies an equation of similar form, namely, $\operatorname{div}\left[\frac{\mathbf{A}^{\prime}\left(y, \lambda^{\prime} \theta^{\prime} v, \lambda^{\prime} \nabla v\right)}{\lambda^{\prime p} p-1}\right]=\operatorname{div} \mathbf{F}^{\prime}$ in $B_{\frac{10}{r}}$ with $\mathbf{A}^{\prime}(y, z, \xi):=\mathbf{A}(r y, z, \xi)$, $\mathbf{F}^{\prime}(y):=\mathbf{F}(r y) / \mu^{p-1}, \lambda^{\prime}:=\mu \lambda$, and $\theta^{\prime}:=r \theta$.

Let us give the precise definition of weak solutions that is used throughout the paper.

Definition 2.3. Let $\mathbf{F} \in L^{p^{\prime}}\left(B_{10} ; \mathbb{R}^{n}\right)$. A function $u \in W_{\text {loc }}^{1, p}\left(B_{10}\right)$ is called a weak solution of (2.4) if $u(x) \in$ $\frac{1}{\lambda \theta} \overline{\mathbb{K}}$ for a.e. $x \in B_{10}$ and

$$
\int_{B_{10}}\left\langle\frac{\mathbf{A}(x, \lambda \theta u, \lambda \nabla u)}{\lambda^{p-1}}, \nabla \varphi\right\rangle d x=\int_{B_{10}}\langle\mathbf{F}, \nabla \varphi\rangle d x \quad \forall \varphi \in W_{0}^{1, p}\left(B_{10}\right) .
$$

Fix a number $M_{0}>0$. Then for a ball $B \subset \mathbb{R}^{n}$, we define

$$
\Theta_{B}(\mathbf{A}):=\sup _{z \in \mathbb{K} \cap\left[-M_{0}, M_{0}\right]} f_{B}\left[\sup _{\xi \neq 0} \frac{\left|\mathbf{A}(x, z, \xi)-\overline{\mathbf{A}}_{B}(z, \xi)\right|}{|\xi|^{p-1}}\right] d x .
$$

In order to achieve the main results stated in Section 1, we will prove the following gradient estimate in weighted $L^{q}$ spaces:

Theorem 2.4. Let A satisfy (1.2)-(1.4) with $p>1$, and let $w$ be an $A_{s}$ weight for some $1<s<\infty$. For any $q \geq p$ and $M_{0}>0$, there exists a constant $\delta=\delta\left(p, q, n, \omega, \Lambda, M_{0}, s,[w]_{A_{s}}\right)>0$ such that: if $\lambda>0, \theta>0$,

$$
\sup _{0<\rho \leq \frac{1}{5}} \sup _{y \in B_{\frac{3}{2}}} \Theta_{B_{\rho}(y)}(\mathbf{A}) \leq \delta
$$

and $u$ is a weak solution of (2.4) satisfying $\|u\|_{L^{\infty}\left(B_{2}\right)} \leq \frac{M_{0}}{\lambda \theta}$, then

$$
f_{B_{1}}|\nabla u|^{q} d w \leq C\left(\|\nabla u\|_{L^{p}\left(B_{2}\right)}^{q}+f_{B_{\frac{3}{2}}} \mathbf{M}_{B_{2}}\left(|\mathbf{F}|^{\frac{p}{p-1}}\right)^{\frac{q}{p}} d w\right) .
$$

Here $C>0$ is a constant depending only on $q, p, n, \omega, \Lambda, M_{0}, s$, and $[w]_{A_{s}}$.

The proof of this key result will be given in Subsection 5.2, and we will demonstrate in Subsection 5.3 that the gradient estimates in weighted Morrey spaces (Theorem 1.2) can be derived as a direct consequence of Theorem 2.4 Notice that by combining Theorem 2.4 with Muckenhoup's strong type weighted estimate for the maximal function (see [26] and [14, Theorem 9.1.9]), we immediately get: 
Corollary 2.5 (weighted $L^{q}$ space estimate). Let A satisfy (1.2)-1.4 with $p>1$. Then for any $q>p$, $M_{0}>0$, and any weight $w \in A_{\frac{q}{p}}$, there exists a constant $\delta>0$ such that: if $\lambda>0, \theta>0$, 2.6 holds, and $u$ is a weak solution of (2.4) satisfying $\|u\|_{L^{\infty}\left(B_{2}\right)} \leq \frac{M_{0}}{\lambda \theta}$, we have

$$
f_{B_{1}}|\nabla u|^{q} d w \leq C\left(\|\nabla u\|_{L^{p}\left(B_{2}\right)}^{q}+f_{B_{2}}|\mathbf{F}|^{\frac{q}{p-1}} d w\right)
$$

Here $C, \delta$ are constants depending only on $q, p, n, \omega, \Lambda, M_{0}$, and $[w]_{A_{q}}$.

\section{Weighted Morrey space estimates for the maximal function}

This section is devoted to deriving a weighted Morrey space estimate for the maximal function. Let us first recall standard maximal operators used in this paper.

Definition 3.1. Let $f \in L_{l o c}^{1}\left(\mathbb{R}^{n}\right)$. The centered Hardy-Littlewood maximal function of $f$ is defined by

$$
\mathrm{M}(f)(x)=\sup _{\rho>0} f_{B_{\rho}(x)}|f(y)| d y .
$$

The uncentered Hardy-Littlewood maximal operator $\tilde{\mathrm{M}}$ is defined over balls by

$$
\tilde{\mathrm{M}}(f)(x)=\sup _{B \ni x} f_{B}|f(y)| d y .
$$

If $U \subset \mathbb{R}^{n}$ is an open set and $f \in L^{1}(U)$, then we denote $\mathrm{M}_{U}(f)=\mathrm{M}\left(f \chi_{U}\right)$ and $\tilde{\mathrm{M}}_{U}(f)=\tilde{\mathrm{M}}\left(f \chi_{U}\right)$.

The following characterization about the two-weight norm inequality

$$
\|\tilde{\mathrm{M}}(f)\|_{L_{w}^{p}\left(\mathbb{R}^{n}\right)} \leq C\|f\|_{L_{v}^{p}\left(\mathbb{R}^{n}\right)}
$$

is due to Sawyer [34] and Kairema [19]:

Theorem 3.2 (two-weight norm inequality). Let $w, v$ be two weights and $1<q<\infty$. Then we have

$$
\|\tilde{\mathrm{M}}(f)\|_{L_{w}^{q}\left(\mathbb{R}^{n}\right)} \leq C_{n} q^{\prime}\left[w, v^{1-q^{\prime}}\right]_{S_{q}}\|f\|_{L_{v}^{q}\left(\mathbb{R}^{n}\right)} \quad \forall f \in L_{v}^{q}\left(\mathbb{R}^{n}\right)
$$

where

$$
[w, \sigma]_{S_{q}}:=\sup _{B}\left(\frac{1}{\sigma(B)} \int_{B}\left[\tilde{\mathrm{M}}\left(\sigma \chi_{B}\right)\right]^{q} w d x\right)^{\frac{1}{q}} .
$$

Moreover, if there exists $C>0$ such that (3.1) holds for all $f \in L_{v}^{q}\left(\mathbb{R}^{n}\right)$ then $\left[w, v^{1-q^{\prime}}\right]_{S_{q}}<\infty$.

Remark 3.3. From the definition of $\tilde{\mathrm{M}}$, it is obvious that

$$
\left[w, v^{1-q^{\prime}}\right]_{A_{q}}:=\sup _{B}\left(f_{B} w(x) d x\right)\left(f_{B} v(x)^{1-q^{\prime}} d x\right)^{q-1} \leq\left[w, v^{1-q^{\prime}}\right]_{S_{q}}^{q} .
$$


Our main result in this subsection is:

Theorem 3.4. Let $w, v$ be two weights and $1<q<\infty$. Assume that $\varphi$ and $\phi$ are two positive functions on the set of nonempty open balls in $\mathbb{R}^{n}$. Then for any bounded open set $U \subset \mathbb{R}^{n}$, we have

$$
\left\|\tilde{\mathbf{M}}_{U}(f)\right\|_{\mathcal{M}_{w}^{q, \varphi}(U)} \leq C(n, q)\left[w, v^{1-q^{\prime}}\right]_{S_{q}}[w \varphi, v \phi]_{q}\|f\|_{\mathcal{M}_{v}^{q, \phi}(U)}
$$

for any function $f \in L^{1}(U)$. Here,

$$
[w \varphi, v \phi]_{q}:=\sup _{y \in U, 0<r \leq \operatorname{diam}(U)}\left[\frac{v\left(B_{2 r}(y)\right)}{w\left(B_{r}(y)\right)} \frac{\phi\left(B_{2 r}(y)\right)^{-1}}{\varphi\left(B_{r}(y)\right)^{-1}}+\sup _{2 r \leq s \leq 2 \operatorname{diam}(U)} \frac{v\left(B_{s}(y)\right)}{w\left(B_{s}(y)\right)} \frac{\phi\left(B_{s}(y)\right)^{-1}}{\varphi\left(B_{r}(y)\right)^{-1}}\right]^{\frac{1}{q}} .
$$

Proof. Without loss of generality, we can assume that $f \in L^{1}(U)$ and $f \geq 0$.

For each $y \in U$ and $0<r \leq \operatorname{diam}(U)$, let us write $f=f \chi_{B_{2 r}(y)}+f \chi_{\mathbb{R}^{n} \backslash B_{2 r}(y)}=: f_{1}+f_{2}$. Then $\tilde{\mathbf{M}}_{U}(f) \leq \tilde{\mathbf{M}}_{U}\left(f_{1}\right)+\tilde{\mathbf{M}}_{U}\left(f_{2}\right)$, and hence

$$
\begin{aligned}
\left\|\tilde{\mathrm{M}}_{U}(f)\right\|_{L_{w}^{q}\left(B_{r}(y) \cap U\right)} & \leq\left\|\tilde{\mathrm{M}}\left(f_{1} \chi_{U}\right)\right\|_{L_{w}^{q}\left(\mathbb{R}^{n}\right)}+\left\|\tilde{\mathrm{M}}\left(f_{2} \chi_{U}\right)\right\|_{L_{w}^{q}\left(B_{r}(y) \cap U\right)} \\
& \leq C_{n} q^{\prime}\left[w, v^{1-q^{\prime}}\right]_{S_{q}}\left\|f_{1} \chi_{U}\right\|_{L_{v}^{q}\left(\mathbb{R}^{n}\right)}+\left\|\tilde{\mathrm{M}}\left(f_{2} \chi_{U}\right)\right\|_{L_{w}^{q}\left(B_{r}(y) \cap U\right)},
\end{aligned}
$$

where we have used Theorem 3.2 to obtain the last inequality. Now for any $x \in B_{r}(y) \cap U$ and any ball $B_{t}(z) \ni x$, we clearly have $B_{t}(z) \subset B_{2 t+r}(y)$. Therefore, by considering separately the case $t \leq r / 2$ and the case $t>r / 2$ we see that

$$
\tilde{\mathbf{M}}\left(\chi_{U} f_{2}\right)(x) \leq 4^{n} \sup _{s>2 r} \frac{1}{\left|B_{s}(y)\right|} \int_{B_{s}(y) \backslash B_{2 r}(y)} f \chi_{U} d z=: 4^{n} I_{r}(y) \quad \forall x \in B_{r}(y) \cap U .
$$

This together with 3.3 yields

$$
\left\|\tilde{\mathbf{M}}_{U}(f)\right\|_{L_{w}^{q}\left(B_{r}(y) \cap U\right)} \leq C_{n} q^{\prime}\left[w, v^{1-q^{\prime}}\right]_{S_{q}}\|f\|_{L_{v}^{q}\left(B_{2 r}(y) \cap U\right)}+4^{n} w\left(B_{r}(y)\right)^{\frac{1}{q}} I_{r}(y)
$$

for every $y \in U$ and $0<r \leq \operatorname{diam}(U)$. Using definition (1.6) and (3.4), we get

$$
\begin{aligned}
\left\|\tilde{\mathbf{M}}_{U}(f)\right\|_{\mathcal{M}_{w}^{q, \varphi}(U)}= & \sup _{y \in U, r \leq \operatorname{diam}(U)}\left(\frac{\varphi\left(B_{r}(y)\right)}{w\left(B_{r}(y)\right)} \int_{B_{r}(y) \cap U}\left|\tilde{\mathbf{M}}_{U}(f)\right|^{q} d w\right)^{\frac{1}{q}} \\
\leq & C_{n} q^{\prime}\left[w, v^{1-q^{\prime}}\right]_{S_{q}} \sup _{y \in U, r \leq \operatorname{diam}(U)}\left(\frac{v\left(B_{2 r}(y)\right)}{w\left(B_{r}(y)\right)} \frac{\varphi\left(B_{r}(y)\right)}{\phi\left(B_{2 r}(y)\right)}\right)^{\frac{1}{q}}\left(\frac{\phi\left(B_{2 r}(y)\right)}{v\left(B_{2 r}(y)\right)} \int_{B_{2 r}(y) \cap U}|f|^{q} d v\right)^{\frac{1}{q}} \\
& +4^{n} \sup _{y \in U, r \leq \operatorname{diam}(U)} \varphi\left(B_{r}(y)\right)^{\frac{1}{q}} I_{r}(y) .
\end{aligned}
$$

When $r \geq \operatorname{diam}(U) / 2$, we have $U \subset B_{2 r}(y)$ and hence $I_{r}(y)=0$. Therefore, we deduce from the above inequality that

$$
\begin{aligned}
\left\|\tilde{\mathrm{M}}_{U}(f)\right\|_{\mathcal{M}_{w}^{q, \varphi}(U) \leq} & C_{n} q^{\prime}\left[w, v^{1-q^{\prime}}\right]_{S_{q}}\|f\|_{\mathcal{M}_{v}^{q, \phi}(U)} \sup _{y \in U, r \leq \operatorname{diam}(U)}\left(\frac{v\left(B_{2 r}(y)\right)}{w\left(B_{r}(y)\right)} \frac{\varphi\left(B_{r}(y)\right)}{\phi\left(B_{2 r}(y)\right)}\right)^{\frac{1}{q}} \\
& +4^{n} \sup _{y \in U, r<\frac{1}{2} \operatorname{diam}(U)} \varphi\left(B_{r}(y)\right)^{\frac{1}{q}} I_{r}(y) .
\end{aligned}
$$


We use Hölder inequality to estimate $I_{r}(y)$ for $r<\frac{1}{2} \operatorname{diam}(U)$ as follows

$$
\begin{aligned}
I_{r}(y) & \leq \sup _{2 r<s} f_{B_{s}(y)} f \chi_{U} d z=\sup _{2 r<s \leq \operatorname{diam}(U)} f_{B_{s}(y)} f \chi_{U} d z \\
& \leq \sup _{2 r<s \leq \operatorname{diam}(U)} \frac{1}{\left|B_{s}(y)\right|}\left(\int_{B_{s}(y)} v^{\frac{-1}{q-1}} d z\right)^{\frac{q-1}{q}}\left(\int_{B_{s}(y)}\left|f \chi_{U}\right|^{q} d v(z)\right)^{\frac{1}{q}} \\
& =\sup _{2 r<s \leq \operatorname{diam}(U)}\left[\left(f_{B_{s}(y)} v d z\right)\left(f_{B_{s}(y)} v^{\frac{-1}{q-1}} d z\right)^{q-1}\right]^{\frac{1}{q}}\left(f_{B_{s}(y)}\left|f \chi_{U}\right|^{q} d v(z)\right)^{\frac{1}{q}} .
\end{aligned}
$$

It then follows from Remark 3.3 that

$$
I_{r}(y) \leq\left[w, v^{1-q^{\prime}}\right]_{S_{q}} \sup _{2 r<s \leq \operatorname{diam}(U)}\left(\frac{v\left(B_{s}(y)\right)}{w\left(B_{s}(y)\right)}\right)^{\frac{1}{q}}\left(f_{B_{s}(y)}\left|f \chi_{U}\right|^{q} d v(z)\right)^{\frac{1}{q}} .
$$

Plugging this into (3.5), we conclude that

$$
\begin{aligned}
& \left\|\tilde{\mathbf{M}}_{U}(f)\right\|_{\mathcal{M}_{w}^{q, \varphi}(U)} \leq C_{n} q^{\prime}\left[w, v^{1-q^{\prime}}\right]_{S_{q}}\|f\|_{\mathcal{M}_{v}^{q, \phi}(U)} \sup _{y \in U, r \leq \operatorname{diam}(U)}\left(\frac{v\left(B_{2 r}(y)\right)}{w\left(B_{r}(y)\right)} \frac{\varphi\left(B_{r}(y)\right)}{\phi\left(B_{2 r}(y)\right)}\right)^{\frac{1}{q}} \\
& \quad+4^{n}\left[w, v^{1-q^{\prime}}\right]_{S_{q}} \sup _{y \in U, r<\frac{1}{2} \operatorname{diam}(U)} \sup _{2 r<s \leq \operatorname{diam}(U)}\left(\frac{v\left(B_{s}(y)\right)}{w\left(B_{s}(y)\right)} \frac{\varphi\left(B_{r}(y)\right)}{\phi\left(B_{s}(y)\right)}\right)^{\frac{1}{q}}\left(\frac{\phi\left(B_{s}(y)\right)}{v\left(B_{s}(y)\right)} \int_{B_{s}(y) \cap U}|f(z)|^{q} d v(z)\right)^{\frac{1}{q}} \\
& \quad \leq C(n, q)\left[w, v^{1-q^{\prime}}\right]_{S_{q}}[w \varphi, v \phi]_{q}\|f\|_{\mathcal{M}_{v}^{q, \phi}(U)}
\end{aligned}
$$

This gives estimate (3.2) as desired.

Remark 3.5. By inspecting the proof we see that inequality (3.2) also holds true if $U$ is replaced by $\mathbb{R}^{n}$.

Given Theorem 3.4, it is desirable to have concrete conditions on $w, v, \varphi$, and $\phi$ ensuring that $\left[w, v^{1-q^{\prime}}\right]_{S_{q}}<$ $\infty$ and $[w \varphi, v \phi]_{q}<\infty$. The finiteness of the constant $\left[w, v^{1-q^{\prime}}\right]_{S_{q}}$ has been investigated extensively (see $[12,28,30,31,38]$ ) and for this to be true it is necessary that $\left[w, v^{1-q^{\prime}}\right]_{A_{q}}<\infty$ (see Remark 3.3). In particular, we have from [30, Corollary 1.3] and [31, Corollary 1.4] that $\left[w, v^{1-q^{\prime}}\right]_{S_{q}}<\infty$ if any of the following two conditions is satisfied:

(A) There exists $r>1$ such that

$$
\sup _{B}\left(f_{B} w d x\right)\left(f_{B} v^{r\left(1-q^{\prime}\right)} d x\right)^{\frac{q-1}{r}}<\infty
$$

(B) $\left[w, v^{1-q^{\prime}}\right]_{A_{q}}<\infty$ and $v^{1-q^{\prime}} \in A_{\infty}$.

We refer readers to [30,31] for more general conditions guaranteeing the finiteness of $\left[w, v^{1-q^{\prime}}\right]_{S_{q}}$. On the other hand, it is clear that $[w \varphi, v \phi]_{q}<\infty$ if $w$ is doubling and there exists a constant $C_{*}>0$ such that

$$
\sup _{2 r \leq s \leq 2 \operatorname{diam}(U)} \frac{v\left(B_{s}(y)\right)}{w\left(B_{s}(y)\right)} \frac{1}{\phi\left(B_{s}(y)\right)} \leq C_{*} \frac{1}{\varphi\left(B_{r}(y)\right)} \quad \text { for all } y \in U \text { and } 0<r \leq \operatorname{diam}(U) .
$$

For convenience, we summarize the above discussions into two separate results: 
Corollary 3.6 (two-weight case). Let $U \subset \mathbb{R}^{n}$ be a bounded open set. Assume that the hypotheses of Theorem 3.4 and condition (3.6) hold, and $w$ is doubling. Assume in addition that either (A) or (B) is satisfied. Then $\left[w, v^{1-q^{\prime}}\right]_{S_{q}}<\infty$ and there exists a constant $C>0$ depending only on $n, q, C_{*}$, the doubling constant for $w$, and $\left[w, v^{1-q^{\prime}}\right]_{S_{q}}$ such that

$$
\left\|\tilde{\mathrm{M}}_{U}(f)\right\|_{\mathcal{M}_{w}^{q, \varphi}(U)} \leq C\|f\|_{\mathcal{M}_{v}^{q, \phi}(U)} \quad \forall f \in L^{1}(U) .
$$

Corollary 3.7 (one-weight case). Let $1<q<\infty, w \in A_{q}$, and $U \subset \mathbb{R}^{n}$ be a bounded open set. Assume that $\varphi$ and $\phi$ are two positive functions on the set of open balls in $\mathbb{R}^{n}$ such that there exists $C_{*}>0$ satisfying

$$
\sup _{2 r \leq s \leq 2 \operatorname{diam}(U)} \phi\left(B_{s}(y)\right)^{-1} \leq C_{*} \varphi\left(B_{r}(y)\right)^{-1} \quad \text { for all } y \in U \text { and } 0<r \leq \operatorname{diam}(U) .
$$

Then there exists a constant $C>0$ depending only on $n, q, C_{*}$, and $[w]_{A_{q}}$ such that

$$
\left\|\tilde{\mathbf{M}}_{U}(f)\right\|_{\mathcal{M}_{w}^{q, \varphi}(U)} \leq C\|f\|_{\mathcal{M}_{w}^{q, \phi}(U)} \text { for any } f \in L^{1}(U) .
$$

Proof. Since $w \in A_{q}$, we have from Lemma 2.1 that $w^{1-q^{\prime}} \in A_{q^{\prime}} \subset A_{\infty}$. Moreover, $\left[w, w^{1-q^{\prime}}\right]_{A_{q}}=[w]_{A_{q}}$. Therefore, the conclusion is a consequence of Corollary 3.6 and the first inequality in 2.1].

Notice that if $\phi=\varphi$, then condition (3.7) trivially holds if $\varphi \in \mathcal{B}_{0}$ defined in Definition 1.1. In particular, we recover the estimate by Chiarenza and Frasca in [5] by taking $\varphi(B):=|B|^{\frac{\lambda}{n}}$ with $0 \leq \lambda \leq n$. Furthermore, Corollary 3.7 extends the weighted Morrey space estimates obtained recently in [15, 16, 21, 27].

\section{Approximating gradients of solutions}

The purpose of this section is to show that gradients of weak solutions to (2.4) can be approximated by bounded gradients in an invariant way. Proposition 4.1] below is an extension of [29, Lemma 5.3] and [2, Lemma 4.2] where the modulus $\omega$ is respectively required to be $\omega(s)=s$ (Lipschitz continuity) and $\omega(s)=s^{\alpha}$ with $\alpha \in(0,1)$ (Hölder continuity). Here by a simple modification of the arguments in [2] we prove that the approximation holds true for any modulus of continuity $\omega$. This result plays a crucial role in our derivation of gradient estimates for solutions of (1.8).

Proposition 4.1. Let $\mathbf{A}$ satisfy (1.2)-(1.4) with $p>1$, and let $M_{0}>0$. For any $\varepsilon \in(0,1]$, there exist small positive constants $\delta$ and $\sigma$ depending only on $\varepsilon, p, n, \omega, \Lambda$, and $M_{0}$ such that: if $\lambda>0, \theta>0, r>0$,

$$
\Theta_{B_{3 \sigma r}}(\mathbf{A}) \leq \delta \quad \text { and } \quad f_{B_{4 r}}|\mathbf{F}|^{p^{\prime}} d x \leq \delta
$$

and $u \in W_{l o c}^{1, p}\left(B_{4 r}\right)$ is a weak solution of $\operatorname{div}\left[\frac{\mathbf{A}(x, \lambda \theta u, \lambda \nabla u)}{\lambda^{p-1}}\right]=\operatorname{div} \mathbf{F}$ in $B_{4 r}$ satisfying

$$
\|u\|_{L^{\infty}\left(B_{4 r}\right)} \leq \frac{M_{0}}{\lambda \theta}, \quad f_{B_{4 r}}|\nabla u|^{p} d x \leq 1, \quad \text { and } \quad f_{B_{4 \sigma r}}|\nabla u|^{p} d x \leq 1
$$

then

$$
f_{B_{3 \sigma r}}|\nabla u-\nabla v|^{p} d x \leq \varepsilon^{p}
$$


for some function $v \in W^{1, p}\left(B_{3 \sigma r}\right)$ with

$$
\|\nabla v\|_{L^{\infty}\left(B_{2 \sigma r}\right)}^{p} \leq C(p, n, \Lambda) f_{B_{3 \sigma r}}|\nabla v|^{p} d x .
$$

Remark 4.2. Since the class of our equations is invariant under the transformation $x \mapsto x+y$, Proposition 4.1 still holds true if $B_{r}$ is replaced by $B_{r}(y)$.

We will use the fact (see the proof of [35, Lemma 1]) that condition (1.2) implies that

$$
\langle\mathbf{A}(x, z, \xi)-\mathbf{A}(x, z, \eta), \xi-\eta\rangle \geq \begin{cases}4^{1-p} \Lambda^{-1}|\xi-\eta|^{p} & \text { if } \quad p \geq 2, \\ 4^{-1} \Lambda^{-1}(|\xi|+|\eta|)^{p-2}|\xi-\eta|^{2} & \text { if } 1<p<2\end{cases}
$$

for a.e. $x \in B_{10}$, all $z \in \overline{\mathbb{K}}$, and all $\xi, \eta \in \mathbb{R}^{n}$. The next result is the first step of proving Proposition 4.1.

Lemma 4.3. For any $\varepsilon>0$, there exist small positive constants $\delta$ and $\sigma$ depending only on $\varepsilon, p, n, \omega, \Lambda$, and $M_{0}$ such that: if $\lambda>0, \theta>0$,

$$
f_{B_{4}}|\mathbf{F}|^{p^{\prime}} d x \leq \delta
$$

and $u \in W_{l o c}^{1, p}\left(B_{4}\right)$ is a weak solution of $\operatorname{div}\left[\frac{\mathbf{A}(x, \lambda \theta u, \lambda \nabla u)}{\lambda^{p-1}}\right]=\operatorname{div} \mathbf{F}$ in $B_{4}$ satisfying

$$
\|u\|_{L^{\infty}\left(B_{4}\right)} \leq \frac{M_{0}}{\lambda \theta} \quad \text { and } \quad f_{B_{4}}|\nabla u|^{p} d x \leq 1
$$

then

$$
f_{B_{4 \sigma}}|\nabla u-\nabla f|^{p} d x \leq \varepsilon^{p}
$$

where $f$ is a weak solution of

$$
\begin{cases}\operatorname{div}\left[\frac{\mathbf{A}\left(x, \lambda \theta \bar{h}_{B_{4 \sigma}}, \lambda \nabla f\right)}{\lambda^{p-1}}\right]=0 & \text { in } \quad B_{4 \sigma} \\ f=h & \text { on } \quad \partial B_{4 \sigma}\end{cases}
$$

with $\bar{h}_{B_{4 \sigma}}:=f_{B_{4 \sigma}} h(x) d x$ and $h$ being a weak solution of

$$
\begin{cases}\operatorname{div}\left[\frac{\mathbf{A}(x, \lambda \theta u, \lambda \nabla h)}{\lambda^{p-1}}\right]=0 & \text { in } B_{4}, \\ h=u & \text { on } \quad \partial B_{4} .\end{cases}
$$

The proof of this result relies on the following classical regularity result for weak solutions of homogeneous equation (4.3) (see for instance [13, estimates (6.64) and (7.45)]).

Lemma 4.4 (classical interior regularity). Assume that $\mathbf{a}(x, \xi)$ satisfies (1.2)-(1.3) with $p>1$. Let $R \in(0,10)$ and $w \in W^{1, p}\left(B_{R}\right)$ be a weak solution of $\operatorname{div} \mathbf{a}(x, \nabla w)=0$ in $B_{R}$. Then there exist constants $p_{0} \in(p, \infty)$, $\beta \in(0,1)$, and $C>0$ depending only on $p, n$, and $\Lambda$ such that for every $r \in(0, R)$ we have

$$
\left(f_{B_{r}}|\nabla w|^{p_{0}} d x\right)^{\frac{1}{p_{0}}} \leq C\left(f_{B_{R}}|\nabla w|^{p} d x\right)^{\frac{1}{p}} \quad \text { and } \quad \sup _{x \in B_{r}}\left|w(x)-\bar{w}_{B_{r}}\right| \leq C\left(\frac{r}{R}\right)^{\beta}\|w\|_{L^{\infty}\left(B_{R}\right)} .
$$


Proof of Lemma 4.3. We present the complete proof only for the case $p \geq 2$ by using an idea in [2], and will indicate at the end how the argument can be modified for the case $1<p<2$. Consider $p \geq 2$ and, for convenience, let $\tilde{\mathbf{A}}(x, z, \xi):=\frac{\mathbf{A}(x, \lambda \theta z, \lambda \xi)}{\lambda^{p-1}}$. We write

$$
\nabla u-\nabla f=\nabla(u-h)+\nabla(h-f)
$$

and will estimate $\|\nabla(u-h)\|_{L^{p}\left(B_{4 \sigma}\right)}$ and $\|\nabla(h-f)\|_{L^{p}\left(B_{4 \sigma}\right)}$. By using $u-h$ as a test function in the equations for $u$ and $h$ we have

$$
\int_{B_{4}}\langle\tilde{\mathbf{A}}(x, u, \nabla u)-\tilde{\mathbf{A}}(x, u, \nabla h), \nabla(u-h)\rangle d x=\int_{B_{4}}\langle\mathbf{F}, \nabla(u-h)\rangle d x .
$$

We then use (4.1) to bound the above left hand side from below. As a consequence, we obtain

$$
\int_{B_{4}}|\nabla(u-h)|^{p} d x \leq 4^{p-1} \Lambda \int_{B_{4}}\langle\mathbf{F}, \nabla(u-h)\rangle d x
$$

Then we infer from Young's inequality that

$$
\int_{B_{4}}|\nabla(u-h)|^{p} d x \leq C \int_{B_{4}}|\mathbf{F}|^{p^{\prime}} d x
$$

yielding

$$
f_{B_{4 \sigma}}|\nabla(u-h)|^{p} d x \leq \frac{C}{\sigma^{n}} f_{B_{4}}|\mathbf{F}|^{p^{\prime}} d x .
$$

By using $h-f$ as a test function in the equations for $h$ and $f$ we have

$$
\int_{B_{4 \sigma}}\left\langle\tilde{\mathbf{A}}\left(x, \bar{h}_{B_{4 \sigma}}, \nabla f\right), \nabla(h-f)\right\rangle d x=\int_{B_{4 \sigma}}\langle\tilde{\mathbf{A}}(x, u, \nabla h), \nabla(h-f)\rangle d x .
$$

This together with (4.1) gives

$$
\begin{aligned}
\int_{B_{4 \sigma}}|\nabla(h-f)|^{p} d x & \leq 4^{p-1} \Lambda \int_{B_{4 \sigma}}\left\langle\tilde{\mathbf{A}}\left(x, \bar{h}_{B_{4 \sigma}}, \nabla h\right)-\tilde{\mathbf{A}}\left(x, \bar{h}_{B_{4 \sigma}}, \nabla f\right), \nabla(h-f)\right\rangle d x \\
& =4^{p-1} \Lambda \int_{B_{4 \sigma}}\left\langle\tilde{\mathbf{A}}\left(x, \bar{h}_{B_{4 \sigma}}, \nabla h\right)-\tilde{\mathbf{A}}(x, u, \nabla h), \nabla(h-f)\right\rangle d x \\
& \leq 4^{p-1} \Lambda \int_{B_{4 \sigma}} \min \left\{2 \Lambda, \omega\left(\lambda \theta\left|u-\bar{h}_{B_{4 \sigma}}\right|\right)\right\}|\nabla h|^{p-1}|\nabla(h-f)| d x .
\end{aligned}
$$

As a consequence of (4.6) and Young's inequality, we obtain

$$
\int_{B_{4 \sigma}}|\nabla(h-f)|^{p} d x \leq C(p, \Lambda) \int_{B_{4 \sigma}}|\nabla h|^{p} d x .
$$

On the other hand, it follows from equation (4.3) for $h$ that $\int_{B_{4 \sigma}}|\nabla h|^{p} d x \leq C \int_{B_{4}}|h|^{p} d x$. Thus we conclude that

$$
f_{B_{4 \sigma}}|\nabla(h-f)|^{p} d x \leq \frac{C}{\sigma^{n}} \int_{B_{4}}|h|^{p} d x \leq \frac{C_{*}}{\sigma^{n}}\|h\|_{L^{\infty}\left(B_{4}\right)}^{p} \leq \frac{C_{*}}{\sigma^{n}}\|u\|_{L^{\infty}\left(B_{4}\right)}^{p} \leq \frac{C_{*}}{\sigma^{n}}\left(\frac{M_{0}}{\lambda \theta}\right)^{p} .
$$


This together with (4.5) gives the desired conclusion if $\frac{C_{*}}{\sigma^{n}}\left(\frac{M_{0}}{\lambda \theta}\right)^{p} \leq \varepsilon^{p} / 2$. We hence only need to consider the case

$$
\frac{C_{*}}{\sigma^{n}}\left(\frac{M_{0}}{\lambda \theta}\right)^{p}>\frac{\varepsilon^{p}}{2} \text {. }
$$

For this, note first that (4.4) and the assumption yield

$$
\|\nabla h\|_{L^{p}\left(B_{4}\right)} \leq\|\nabla(h-u)\|_{L^{p}\left(B_{4}\right)}+\|\nabla u\|_{L^{p}\left(B_{4}\right)} \leq C\left[\left(\int_{B_{4}}|\mathbf{F}|^{p^{\prime}} d x\right)^{\frac{1}{p}}+1\right] \leq C .
$$

We deduce from (4.6), Young's inequality, the higher integrability for $\nabla h$ given by Lemma 4.4, and (4.8) that

$$
\begin{aligned}
f_{B_{4 \sigma}}|\nabla(h-f)|^{p} d x & \leq C f_{B_{4 \sigma}} \omega\left(\lambda \theta\left|u-\bar{h}_{B_{4 \sigma}}\right|\right)^{p^{\prime}}|\nabla h|^{p} d x \\
& \leq C\left[f_{B_{4 \sigma}} \omega\left(\lambda \theta\left|u-\bar{h}_{B_{4 \sigma}}\right|\right)^{\frac{p^{\prime} p_{0}}{p_{0}-p}} d x\right]^{\frac{p_{0}-p}{p_{0}}}\left[f_{B_{4 \sigma}}|\nabla h|^{p_{0}} d x\right]^{\frac{p}{p_{0}}} \\
& \leq C\left[f_{B_{4 \sigma}} \omega\left(\lambda \theta\left|u-\bar{h}_{B_{4 \sigma}}\right|\right)^{\frac{p^{\prime} p_{0}}{p_{0}-p}} d x\right]^{\frac{p_{0}-p}{p_{0}}} f_{B_{4}}|\nabla h|^{p} d x \leq C\left[f_{B_{4 \sigma}} \omega\left(\lambda \theta\left|u-\bar{h}_{B_{4 \sigma}}\right|\right)^{\frac{p^{\prime} p_{0}}{p_{0}-p}} d x\right]^{\frac{p_{0}-p}{p_{0}}}
\end{aligned}
$$

with $p_{0}>p$ and $C>0$ depending only on $p, n$, and $\Lambda$. As for any $\gamma>0$, we have

$$
\begin{aligned}
\int_{B_{4 \sigma}} \omega\left(\lambda \theta\left|u-\bar{h}_{B_{4 \sigma}}\right|\right)^{\frac{p^{\prime} p_{0}}{p_{0}-p}} d x & =\int_{\left\{B_{4 \sigma}: \lambda \theta\left|u-\bar{h}_{B_{4 \sigma}}\right| \leq \gamma\right\}} \omega\left(\lambda \theta\left|u-\bar{h}_{B_{4 \sigma}}\right|\right)^{\frac{p^{\prime} p_{0}}{p_{0}-p}} d x \\
& +\int_{\left\{B_{4 \sigma}: \lambda \theta\left|u-\bar{h}_{B_{4 \sigma}}\right|>\gamma\right\}} \omega\left(\lambda \theta\left|u-\bar{h}_{B_{4 \sigma}}\right|\right)^{\frac{p^{\prime} p_{0}}{p_{0}-p}} d x \\
& \leq\left|B_{4 \sigma}\right| \omega(\gamma)^{\frac{p^{\prime} p_{0}}{p_{0}-p}}+\frac{\omega\left(2 M_{0}\right)^{\frac{p^{\prime} p_{0}}{p_{0}-p}}}{\gamma^{p}} \int_{B_{4 \sigma}}\left(\lambda \theta\left|u-\bar{h}_{B_{4 \sigma}}\right|\right)^{p} d x,
\end{aligned}
$$

we infer that

$$
f_{B_{4 \sigma}}|\nabla(h-f)|^{p} d x \leq C \omega(\gamma)^{p^{\prime}}+C \frac{\omega\left(2 M_{0}\right)^{p^{\prime}}}{\gamma^{\frac{p\left(p_{0}-p\right)}{p_{0}}}}\left[(\lambda \theta)^{p} f_{B_{4 \sigma}}\left|u-\bar{h}_{B_{4 \sigma}}\right|^{p} d x\right]^{\frac{p_{0}-p}{p_{0}}} \quad \forall \gamma>0 .
$$

Let us now estimate the last integral in 4.97. Using Sobolev's inequality and the oscillation estimate in Lemma 4.4, we have

$$
\begin{aligned}
f_{B_{4 \sigma}}\left|u-\bar{h}_{B_{4 \sigma}}\right|^{p} d x & \leq 2^{p-1}\left[\frac{1}{\left|B_{4 \sigma}\right|} \int_{B_{4}}|u-h|^{p} d x+f_{B_{4 \sigma}}\left|h-\bar{h}_{B_{4 \sigma}}\right|^{p} d x\right] \\
& \leq C\left[\sigma^{-n} \int_{B_{4}}|\nabla(u-h)|^{p} d x+\left(\sigma^{\beta}\|h\|_{L^{\infty}\left(B_{4}\right)}\right)^{p}\right] .
\end{aligned}
$$

This together with (4.7) and the fact $\|h\|_{L^{\infty}\left(B_{4}\right)} \leq M_{0} / \lambda \theta$ yields

$$
(\lambda \theta)^{p} f_{B_{4 \sigma}}\left|u-\bar{h}_{B_{4 \sigma}}\right|^{p} d x \leq C\left[\sigma^{-2 n}\left(\frac{M_{0}}{\varepsilon}\right)^{p} \int_{B_{4}}|\nabla(u-h)|^{p} d x+\left(M_{0} \sigma^{\beta}\right)^{p}\right] .
$$


Plugging this estimate into (4.9) gives

$$
f_{B_{4 \sigma}}|\nabla(h-f)|^{p} d x \leq C \omega(\gamma)^{p^{\prime}}+C M_{0}^{\frac{p\left(p_{0}-p\right)}{p_{0}}} \omega\left(2 M_{0}\right)^{p^{\prime}}\left[\frac{\sigma^{-2 n}}{(\gamma \varepsilon)^{p}} \int_{B_{4}}|\nabla(u-h)|^{p} d x+\left(\frac{\sigma^{\beta}}{\gamma}\right)^{p}\right]^{\frac{p_{0}-p}{p_{0}}} .
$$

By combining this with (4.5) and using (4.4) we obtain

$$
\begin{aligned}
f_{B_{4 \sigma}}|\nabla(u-f)|^{p} d x \leq & \frac{C}{\sigma^{n}} f_{B_{4}}|\mathbf{F}|^{p^{\prime}} d x \\
& +C \omega(\gamma)^{p^{\prime}}+C M_{0}^{\frac{p\left(p_{0}-p\right)}{p_{0}}} \omega\left(2 M_{0}\right)^{p^{\prime}}\left[\frac{\sigma^{-2 n}}{(\gamma \varepsilon)^{p}} f_{B_{4}}|\mathbf{F}|^{p^{\prime}} d x+\left(\frac{\sigma^{\beta}}{\gamma}\right)^{p}\right]^{\frac{p_{0}-p}{p_{0}}}
\end{aligned}
$$

for every $\gamma>0$. From this, we get the desired conclusion by choosing $\gamma$ small first, then $\sigma$, and $\delta$ last. For the case $1<p<2$, the proof is similar with some slight adjustments as follows, which can also be found in the proofs of Lemma 4.3 and Lemma 4.6 in [2]. By arguing as above but using (4.1] together with [29, Lemma 3.1], then in place of (4.5) and (4.6) we now have for every $\tau_{1}, \tau_{2}>0$ small that

$$
f_{B_{4 \sigma}}|\nabla(u-h)|^{p} d x \leq \frac{C}{\sigma^{n}}\left[\tau_{1} f_{B_{4}}|\nabla u|^{p} d x+\tau_{1}^{\frac{p-2}{p-1}} f_{B_{4}}|\mathbf{F}|^{p^{\prime}} d x\right] \leq \frac{C}{\sigma^{n}}\left[\tau_{1}+\tau_{1}^{\frac{p-2}{p-1}} f_{B_{4}}|\mathbf{F}|^{p^{\prime}} d x\right]
$$

and

$$
\begin{aligned}
\int_{B_{4 \sigma}}|\nabla(h-f)|^{p} d x & \leq \tau_{2} \int_{B_{4 \sigma}}|\nabla h|^{p} d x+C_{p} \Lambda \tau_{2}^{1-\frac{2}{p}} \int_{B_{4 \sigma}}\left\langle\tilde{\mathbf{A}}\left(x, \bar{h}_{B_{4 \sigma}}, \nabla h\right)-\tilde{\mathbf{A}}\left(x, \bar{h}_{B_{4 \sigma}}, \nabla f\right), \nabla(h-f)\right\rangle d x \\
& \leq \tau_{2} \int_{B_{4 \sigma}}|\nabla h|^{p} d x+C_{p} \Lambda \tau_{2}^{1-\frac{2}{p}} \int_{B_{4 \sigma}} \min \left\{2 \Lambda, \omega\left(\lambda \theta\left|u-\bar{h}_{B_{4 \sigma}}\right|\right)\right\}|\nabla h|^{p-1}|\nabla(h-f)| d x .
\end{aligned}
$$

With these changes and by repeating the above lines of argument, we obtain the following version of (4.10) for the case $1<p<2$ :

$$
\begin{aligned}
f_{B_{4 \sigma}}|\nabla(u-f)|^{p} d x \leq & \frac{C}{\sigma^{n}}\left[\tau_{1}+\tau_{1}^{\frac{p-2}{p-1}} f_{B_{4}}|\mathbf{F}|^{p^{\prime}} d x\right] \\
& +C \tau_{2}+C \tau_{2}^{\frac{p-2}{p-1}}\left\{\omega(\gamma)^{p^{\prime}}+M_{0}^{\frac{p\left(p_{0}-p\right)}{p_{0}}} \omega\left(2 M_{0}\right)^{p^{\prime}}\left[\frac{\sigma^{-2 n}}{(\gamma \varepsilon)^{p}} f_{B_{4}}|\mathbf{F}|^{p^{\prime}} d x+\left(\frac{\sigma^{\beta}}{\gamma}\right)^{p}\right]^{\frac{p_{0}-p}{p_{0}}}\right\} .
\end{aligned}
$$

We then get the conclusion by choosing $\tau_{2}$ small first, $\gamma$ second, $\sigma$ third, then $\tau_{1}$, and $\delta$ last.

To obtain Proposition 4.1, our second step is to show that the gradient of the solution $f$ to (4.2) can be approximated by a bounded gradient. Precisely, we have:

Lemma 4.5. Let $\varepsilon \in(0,1]$, and let $\sigma$ be its corresponding constant given by Lemma 4.3 Let $\mathbf{F}, u$, and $h$ be as in Lemma 4.3 and assume in addition that $f_{B_{4 \sigma}}|\nabla u|^{p} d x \leq 1$. Suppose that $f$ is a weak solution of $\operatorname{div}\left[\frac{\mathbf{A}\left(x, \lambda \theta \bar{h}_{B_{4 \sigma}}, \lambda \nabla f\right)}{\lambda^{p-1}}\right]=0$ in $B_{4 \sigma}$ and $v$ is a weak solution of

$$
\begin{cases}\operatorname{div}\left[\frac{\overline{\mathbf{A}}_{B_{3 \sigma}}\left(\lambda \theta \bar{h}_{B_{4 \sigma}}, \lambda \nabla v\right)}{\lambda^{p-1}}\right]=0 & \text { in } \quad B_{3 \sigma} \\ v=f & \text { on } \quad \partial B_{3 \sigma}\end{cases}
$$


There exist constants $p_{0} \in(p, \infty)$ and $C>0$ depending only on $p, n$, and $\Lambda$ such that: if $p \geq 2$, then

$$
f_{B_{3 \sigma}}|\nabla f-\nabla v|^{p} d x \leq C \Theta_{B_{3 \sigma}}(\mathbf{A})^{\frac{p_{0}-p}{p_{0}}}
$$

and if $1<p<2$, then

$$
f_{B_{3 \sigma}}|\nabla f-\nabla v|^{p} d x \leq \tau+C \tau^{\left(1-\frac{2}{p}\right) p^{\prime}} \Theta_{B_{3 \sigma}}(\mathbf{A})^{\frac{p_{0}-p}{p_{0}}} \quad \text { for every } \quad \tau \in(0,1) .
$$

Proof. For convenience, define $\mathbf{a}(x, \xi):=\frac{\mathbf{A}\left(x, \lambda \theta \bar{h}_{B_{G \sigma}}, \lambda \xi\right)}{\lambda^{p-1}}$ and $\overline{\mathbf{a}}_{B_{3 \sigma}}(\xi):=f_{B_{3 \sigma}} \mathbf{a}(x, \xi) d x$. We first consider the case $p \geq 2$. Then using (4.1) we get

$$
\int_{B_{3 \sigma}}|\nabla(f-v)|^{p} d x \leq \Lambda \int_{B_{3 \sigma}}\left\langle\overline{\mathbf{a}}_{B_{3 \sigma}}(\nabla f)-\overline{\mathbf{a}}_{B_{3 \sigma}}(\nabla v), \nabla(f-v)\right\rangle d x:=I .
$$

To estimate the term $I$, we use $f-v$ as a test function in equation (4.11) and the equation for $f$ to obtain

$$
\int_{B_{3 \sigma}}\left\langle\overline{\mathbf{a}}_{B_{3 \sigma}}(\nabla v), \nabla(f-v)\right\rangle d x=\int_{B_{3 \sigma}}\langle\mathbf{a}(x, \nabla f), \nabla(f-v)\rangle d x .
$$

Therefore,

$$
I=\Lambda \int_{B_{3 \sigma}}\left\langle\overline{\mathbf{a}}_{B_{3 \sigma}}(\nabla f)-\mathbf{a}(x, \nabla f), \nabla(f-v)\right\rangle d x \leq \Lambda \int_{B_{3 \sigma}} \sup _{\xi \neq 0} \frac{\left|\mathbf{a}(x, \xi)-\overline{\mathbf{a}}_{B_{3 \sigma}}(\xi)\right|}{|\xi|^{p-1}}|\nabla f|^{p-1}|\nabla(f-v)| d x .
$$

It follows from (4.13)-4.14), Young's inequality, the interior higher integrability for $\nabla f$ given by Lemma 4.4, and the fact $|\mathbf{a}(x, \xi)| \leq \Lambda|\xi|^{p-1}$ that

$$
\begin{aligned}
f_{B_{3 \sigma}}|\nabla(f-v)|^{p} d x & \leq C f_{B_{3 \sigma}}\left[\sup _{\xi \neq 0} \frac{\left|\mathbf{a}(x, \xi)-\overline{\mathbf{a}}_{B_{3 \sigma}}(\xi)\right|}{|\xi|^{p-1}}\right]^{p^{\prime}}|\nabla f|^{p} d x \\
& \leq C\left(f_{B_{3 \sigma}}\left[\sup _{\xi \neq 0} \frac{\left|\mathbf{a}(x, \xi)-\overline{\mathbf{a}}_{B_{3 \sigma}}(\xi)\right|}{|\xi|^{p-1}}\right]^{\frac{p^{\prime} p_{0}}{p_{0}-p}} d x\right)^{\frac{p_{0}-p}{p_{0}}}\left(f_{B_{3 \sigma}}|\nabla f|^{p_{0}} d x\right)^{\frac{p}{p_{0}}} \\
& \leq C\left(f_{B_{3 \sigma}} \sup _{\xi \neq 0} \frac{\left|\mathbf{a}(x, \xi)-\overline{\mathbf{a}}_{B_{3 \sigma}}(\xi)\right|}{|\xi|^{p-1}} d x\right)^{\frac{p_{0}-p}{p_{0}}} f_{B_{4 \sigma}}|\nabla f|^{p} d x
\end{aligned}
$$

where $C>0$ depends only on $p, n$, and $\Lambda$. But we have from the definition of a that

$$
\sup _{\xi \neq 0} \frac{\left|\mathbf{a}(x, \xi)-\overline{\mathbf{a}}_{B_{3 \sigma}}(\xi)\right|}{|\xi|^{p-1}}=\sup _{\eta \neq 0} \frac{\left|\mathbf{A}\left(x, \lambda \theta \bar{h}_{B_{4 \sigma}}, \eta\right)-\overline{\mathbf{A}}_{B_{3 \sigma}}\left(\lambda \theta \bar{h}_{B_{4 \sigma}}, \eta\right)\right|}{|\eta|^{p-1}}
$$

and Lemma 4.3 yields

$$
f_{B_{4 \sigma}}|\nabla f|^{p} d x \leq 2^{p-1}\left[f_{B_{4 \sigma}}|\nabla f-\nabla u|^{p} d x+f_{B_{4 \sigma}}|\nabla u|^{p} d x\right] \leq 2^{p-1}\left[\varepsilon^{p}+1\right] \leq 2^{p} .
$$


Therefore we conclude that

$$
f_{B_{3 \sigma}}|\nabla(f-v)|^{p} d x \leq C\left(f_{B_{3 \sigma}} \sup _{\xi \neq 0} \frac{\left|\mathbf{A}\left(x, \lambda \theta \bar{h}_{B_{4 \sigma}}, \xi\right)-\overline{\mathbf{A}}_{B_{3 \sigma}}\left(\lambda \theta \bar{h}_{B_{4 \sigma}}, \xi\right)\right|}{|\xi|^{p-1}} d x\right)^{\frac{p_{0}-p}{p_{0}}} .
$$

This together with the fact $\lambda \theta \bar{h}_{B_{4 \sigma}} \in \overline{\mathbb{K}} \cap\left[-M_{0}, M_{0}\right]$ and the definition of $\Theta_{B_{3 \sigma}}$ (A) given by (2.5) yields estimate (4.12). We next consider the case $1<p<2$. Then condition (1.2) in [29] is satisfied thanks to (4.1). Therefore, instead of (4.13) we now have from [29, Lemma 3.1] that

$$
\int_{B_{3 \sigma}}|\nabla(f-v)|^{p} d x \leq \tau \int_{B_{3 \sigma}}|\nabla f|^{p} d x+C_{p} \tau^{1-\frac{2}{p}} I \quad \text { for all } \quad \tau \in\left(0, \frac{1}{2}\right) .
$$

Then we deduce from estimate (4.14) for $I$ and Young's inequality that

$$
f_{B_{3 \sigma}}|\nabla(f-v)|^{p} d x \leq 2 \tau \int_{B_{3 \sigma}}|\nabla f|^{p} d x+C(p, \Lambda) \tau^{\left(1-\frac{2}{p}\right) p^{\prime}} f_{B_{3 \sigma}}\left[\sup _{\xi \neq 0} \frac{\left|\mathbf{a}(x, \xi)-\overline{\mathbf{a}}_{B_{3 \sigma}}(\xi)\right|}{|\xi|^{p-1}}\right]^{p^{\prime}}|\nabla f|^{p} d x .
$$

The first integral is estimated by (4.15) and the last integral can be estimated exactly as above. As a consequence, we obtain

$$
f_{B_{3 \sigma}}|\nabla(f-v)|^{p} d x \leq 2^{p+1}\left(\frac{4}{3}\right)^{n} \tau+C(p, n, \Lambda) \tau^{\left(1-\frac{2}{p}\right) p^{\prime}} \Theta_{B_{3 \sigma}}(\mathbf{A})^{\frac{p_{0}-p}{p_{0}}} \quad \text { for all } \quad \tau \in\left(0, \frac{1}{2}\right) .
$$

We are now ready to prove Proposition 4.1 .

Proof of Proposition 4.1, The stated result follows by scaling and then applying Lemma 4.3 and Lemma 4.5 Precisely, let us define

$$
\mathbf{A}^{\prime}(x, z, \xi)=\mathbf{A}(r x, z, \xi), \quad \mathbf{F}^{\prime}(x)=\mathbf{F}(r x), \quad u^{\prime}(x)=r^{-1} u(r x), \quad \text { and } \theta^{\prime}=\theta r .
$$

Then $u^{\prime}$ is a weak solution of $\operatorname{div}\left[\frac{\mathbf{A}^{\prime}\left(x, \lambda \theta^{\prime} u^{\prime}, \lambda \nabla u^{\prime}\right)}{\lambda^{p-1}}\right]=\operatorname{div} \mathbf{F}^{\prime}$ in $B_{4}$ satisfying

$$
\left\|u^{\prime}\right\|_{L^{\infty}\left(B_{4}\right)} \leq \frac{M_{0}}{\lambda \theta^{\prime}}, \quad f_{B_{4}}\left|\nabla u^{\prime}\right|^{p} d x=f_{B_{4 r}}|\nabla u|^{p} d y \leq 1, \quad f_{B_{4 \sigma}}\left|\nabla u^{\prime}\right|^{p} d x=f_{B_{4 \sigma r}}|\nabla u|^{p} d y \leq 1 .
$$

We also have

$$
\Theta_{B_{3 \sigma}}\left(\mathbf{A}^{\prime}\right)=\Theta_{B_{3 \sigma r}}(\mathbf{A}) \leq \delta \quad \text { and } \quad f_{B_{4}}\left|\mathbf{F}^{\prime}\right|^{p^{\prime}} d x=f_{B_{4 r}}|\mathbf{F}|^{p^{\prime}} d y \leq \delta .
$$

Therefore, we can apply Lemma 4.3 and Lemma 4.5 to obtain that

$$
f_{B_{3 \sigma}}\left|\nabla u^{\prime}-\nabla v^{\prime}\right|^{p} d x \leq \varepsilon^{p}
$$

where $v^{\prime}$ is a weak solution of

$$
\operatorname{div}\left[\frac{\overline{\mathbf{A}}_{B_{\sigma}}^{\prime}\left(\lambda \theta^{\prime} \bar{h}_{B_{4 \sigma}}, \lambda \nabla v^{\prime}\right)}{\lambda^{p-1}}\right]=0 \quad \text { in } \quad B_{3 \sigma}
$$


From the well known interior Lipschitz estimate for this constant coefficient equation we have

$$
\left\|\nabla v^{\prime}\right\|_{L^{\infty}\left(B_{2 \sigma}\right)}^{p} \leq C(p, n, \Lambda) f_{B_{3 \sigma}}\left|\nabla v^{\prime}\right|^{p} d x .
$$

Let $v(y):=r v^{\prime}\left(r^{-1} y\right)$. Then by rescaling we obtain from (4.16) and (4.17) that

$$
f_{B_{3 \sigma r}}|\nabla u-\nabla v|^{p} d y \leq \varepsilon^{p} \quad \text { and } \quad\|\nabla v\|_{L^{\infty}\left(B_{2 \sigma r}\right)}^{p} \leq C(p, n, \Lambda) f_{B_{3 \sigma r}}|\nabla v|^{p} d y .
$$

\section{Density and gradient estimates}

We derive interior gradient estimates for weak solution $u$ of (2.4) by estimating the distribution functions of the maximal function of $|\nabla u|^{p}$. This is carried out in the next two subsections, while the last subsection (Subsection 5.3) is devoted to proving the main results stated in Section1.

\subsection{Density estimates}

The next result gives a density estimate for the distribution of $\mathrm{M}_{B_{1}}\left(|\nabla u|^{p}\right)$. It roughly says that if the maximal function $\mathrm{M}_{B_{1}}\left(|\nabla u|^{p}\right)$ is bounded at one point in $B_{\sigma r}(y)$ then this property can be propagated for all points in $B_{\sigma r}(y)$ except on a set of small measure $w$.

Lemma 5.1. Assume that $\mathbf{A}$ satisfies (1.2)-(1.4) and $\mathbf{F} \in L^{p^{\prime}}\left(B_{10} ; \mathbb{R}^{n}\right)$. Let $M_{0}>0$ and $w$ be an $A_{\infty}$ weight. There exists a constant $N=N(p, n, \Lambda)>1$ satisfying for any $\varepsilon>0$, we can find small positive constants $\delta$ and $\sigma$ depending only on $\varepsilon, p, n, \omega, \Lambda, M_{0}$, and $[w]_{A_{\infty}}$ such that: if $\lambda>0, \theta>0$,

$$
\sup _{0<\rho \leq \frac{3}{5}} \sup _{y \in B_{\frac{\sigma}{10}}} \Theta_{B_{\sigma \rho}(y)}(\mathbf{A}) \leq \delta,
$$

then for any weak solution $u$ of (2.4) with $\|u\|_{L^{\infty}\left(B_{1}\right)} \leq \frac{M_{0}}{\lambda \theta}$, and for any $y \in B_{\frac{\sigma}{10}}, 0<r \leq \frac{1}{5}$ with

$$
B_{\sigma r}(y) \cap B_{\frac{\sigma}{10}} \cap\left\{B_{1}: \mathrm{M}_{B_{1}}\left(|\nabla u|^{p}\right) \leq 1\right\} \cap\left\{B_{1}: \mathrm{M}_{B_{1}}\left(|\mathbf{F}|^{p^{\prime}}\right) \leq \delta\right\} \neq \emptyset,
$$

we have

$$
w\left(\left\{B_{\frac{\sigma}{10}}: \mathrm{M}_{B_{1}}\left(|\nabla u|^{p}\right)>N\right\} \cap B_{\sigma r}(y)\right)<\varepsilon w\left(B_{\sigma r}(y)\right) .
$$

Proof. By (5.1) there exists $x_{0} \in B_{\sigma r}(y) \cap B_{\frac{\sigma}{10}}$ such that

$$
\mathrm{M}_{B_{1}}\left(|\nabla u|^{p}\right)\left(x_{0}\right) \leq 1 \quad \text { and } \quad \mathrm{M}_{B_{1}}\left(|\mathbf{F}|^{p^{\prime}}\right)\left(x_{0}\right) \leq \delta .
$$

This together with the facts $B_{4 r}(y) \subset B_{5 r}\left(x_{0}\right) \cap B_{1}$ and $B_{4 \sigma r}(y) \subset B_{5 \sigma r}\left(x_{0}\right) \cap B_{1}$ implies that

$$
\begin{aligned}
& f_{B_{4 r}(y)}|\nabla u|^{p} d x \leq \frac{\left|B_{5 r}\left(x_{0}\right)\right|}{\left|B_{4 r}(y)\right|} \frac{1}{\left|B_{5 r}\left(x_{0}\right)\right|} \int_{B_{5 r}\left(x_{0}\right) \cap B_{1}}|\nabla u|^{p} d x \leq\left(\frac{5}{4}\right)^{n}, \\
& f_{B_{4 \sigma r}(y)}|\nabla u|^{p} d x \leq \frac{\left|B_{5 \sigma r}\left(x_{0}\right)\right|}{\left|B_{4 \sigma r}(y)\right|} \frac{1}{\left|B_{5 \sigma r}\left(x_{0}\right)\right|} \int_{B_{5 \sigma r}\left(x_{0}\right) \cap B_{1}}|\nabla u|^{p} d x \leq\left(\frac{5}{4}\right)^{n}, \\
& f_{B_{4 r}(y)}|\mathbf{F}|^{p^{\prime}} d x \leq \frac{\left|B_{5 r}\left(x_{0}\right)\right|}{\left|B_{4 r}(y)\right|} \frac{1}{\left|B_{5 r}\left(x_{0}\right)\right|} \int_{B_{5 r}\left(x_{0}\right) \cap B_{1}}|\mathbf{F}|^{p^{\prime}} d x \leq\left(\frac{5}{4}\right)^{n} \delta .
\end{aligned}
$$


Therefore, we can apply Proposition 4.1 and Remark 4.2 for $\tilde{\varepsilon} \in(0,1]$ that will be determined later. As a consequence, we obtain

$$
f_{B_{3 \sigma r}(y)}|\nabla u-\nabla v|^{p} d x \leq \tilde{\varepsilon}^{p}
$$

for some function $v \in W^{1, p}\left(B_{3 \sigma r}(y)\right)$ with

$$
\|\nabla v\|_{L^{\infty}\left(B_{2 \sigma r}(y)\right)}^{p} \leq C(p, n, \Lambda) f_{B_{3 \sigma r}(y)}|\nabla v|^{p} d x .
$$

These combined with the first inequality in (5.2) give

$$
\|\nabla v\|_{L^{\infty}\left(B_{2 \sigma r}(y)\right)}^{p} \leq 2^{p-1} C\left[f_{B_{3 \sigma r}(y)}|\nabla u-\nabla v|^{p} d x+f_{B_{3 \sigma r}(y)}|\nabla u|^{p} d x\right] \leq 2^{p-1} C\left[\tilde{\varepsilon}^{p}+\left(\frac{4}{3}\right)^{n}\right] \leq C_{*},
$$

where $C^{*}>0$ depends only on $p, n$, and $\Lambda$. We claim that (5.2)-(5.4) yield

$$
\left\{B_{\sigma r}(y): \mathrm{M}_{B_{3 \sigma r}(y)}\left(|\nabla u-\nabla v|^{p}\right) \leq C_{*}\right\} \subset\left\{B_{\sigma r}(y): \mathrm{M}_{B_{1}}\left(|\nabla u|^{p}\right) \leq N\right\}
$$

with $N:=\max \left\{2^{p} C_{*}, 3^{n}\right\}$. Indeed, let $x$ be a point in the set on the left hand side of (5.5), and consider $B_{\rho}(x)$. If $\rho \leq \sigma r$, then $B_{\rho}(x) \subset B_{2 \sigma r}(y) \subset B_{1}$ and hence

$$
\begin{aligned}
\frac{1}{\left|B_{\rho}(x)\right|} \int_{B_{\rho}(x) \cap B_{1}}|\nabla u|^{p} d y & \leq \frac{2^{p-1}}{\left|B_{\rho}(x)\right|}\left[\int_{B_{\rho}(x) \cap B_{1}}|\nabla u-\nabla v|^{p} d y+\int_{B_{\rho}(x) \cap B_{1}}|\nabla v|^{p} d y\right] \\
& \leq 2^{p-1}\left[\mathrm{M}_{B_{3 \sigma r}(y)}\left(|\nabla u-\nabla v|^{p}\right)(x)+\|\nabla v\|_{L^{\infty}\left(B_{2 \sigma r}(y)\right)}^{p}\right] \leq 2^{p} C_{*} .
\end{aligned}
$$

On the other hand, if $\rho>\sigma r$ then $B_{\rho}(x) \subset B_{3 \rho}\left(x_{0}\right)$. This and the first inequality in (5.2) give

$$
\frac{1}{\left|B_{\rho}(x)\right|} \int_{B_{\rho}(x) \cap B_{1}}|\nabla u|^{p} d y \leq \frac{3^{n}}{\left|B_{3 \rho}\left(x_{0}\right)\right|} \int_{B_{3 \rho}\left(x_{0}\right) \cap B_{1}}|\nabla u|^{p} d y \leq 3^{n} .
$$

Therefore, $\mathrm{M}_{B_{1}}\left(|\nabla u|^{p}\right)(x) \leq N$ and claim (5.5) is proved. Notice that (5.5) is equivalent to

$$
\left\{B_{\sigma r}(y): \mathrm{M}_{B_{1}}\left(|\nabla u|^{p}\right)>N\right\} \subset\left\{B_{\sigma r}(y): \mathrm{M}_{B_{3 \sigma r}(y)}\left(|\nabla u-\nabla v|^{p}\right)>C_{*}\right\} .
$$

It follows from this, the weak type $1-1$ estimate, and (5.3) that

$$
\begin{aligned}
\left|\left\{B_{\sigma r}(y): \mathrm{M}_{B_{1}}\left(|\nabla u|^{p}\right)>N\right\}\right| & \leq\left|\left\{B_{\sigma r}(y): \mathrm{M}_{B_{3 \sigma r}(y)}\left(|\nabla u-\nabla v|^{p}\right)>C_{*}\right\}\right| \\
& \leq C \int_{B_{3 \sigma r}(y)}|\nabla u-\nabla v|^{p} d x \leq C_{1} \tilde{\varepsilon}^{p}\left|B_{\sigma r}(y)\right| .
\end{aligned}
$$

We then infer from property 2.2 that

$$
w\left(\left\{B_{\sigma r}(y): \mathrm{M}_{B_{1}}\left(|\nabla u|^{p}\right)>N\right\}\right) \leq A\left(\frac{\left|\left\{B_{\sigma r}(y): \mathrm{M}_{B_{1}}\left(|\nabla u|^{p}\right)>N\right\}\right|}{\left|B_{\sigma r}(y)\right|}\right)^{v} w\left(B_{\sigma r}(y)\right) \leq A\left(C_{1} \tilde{\varepsilon}^{p}\right)^{v} w\left(B_{\sigma r}(y)\right)
$$

with $A$ and $v$ being the constants given by characterization (2.2) for $w$. We choose $\tilde{\varepsilon}^{p}:=\min \left\{C_{1}^{-1}\left(\varepsilon A^{-1}\right)^{\frac{1}{v}}, 1\right\}$ to complete the proof. 
In view of Lemma 5.1, we can apply a variation of the Vitali covering lemma as in [25, Lemma 3.8] to obtain:

Lemma 5.2. Assume that $\mathbf{A}$ satisfies (1.2)-(1.4) and $\mathbf{F} \in L^{p^{\prime}}\left(B_{10} ; \mathbb{R}^{n}\right)$. Let $M_{0}>0$ and $w$ be an $A_{s}$ weight for some $1<s<\infty$. There exists a constant $N=N(p, n, \Lambda)>1$ satisfying for any $\varepsilon>0$, we can find small positive constants $\delta$ and $\sigma$ depending only on $\varepsilon, p, n, \omega, \Lambda, M_{0}$, and $[w]_{A_{s}}$ such that: if $\lambda>0, \theta>0$,

$$
\sup _{0<\rho \leq \frac{3}{5}} \sup _{y \in B_{\frac{\sigma}{10}}} \Theta_{B_{\sigma \rho}(y)}(\mathbf{A}) \leq \delta
$$

then for any weak solution $u$ of (2.4) satisfying $\|u\|_{L^{\infty}\left(B_{1}\right)} \leq \frac{M_{0}}{\lambda \theta}$ and

$$
w\left(\left\{B_{\frac{\sigma}{10}}: \mathrm{M}_{B_{1}}\left(|\nabla u|^{p}\right)>N\right\}\right)<\varepsilon w\left(B_{\frac{\sigma}{10}}\right),
$$

we have

$$
w\left(\left\{B_{\frac{\sigma}{10}}: \mathbf{M}_{B_{1}}\left(|\nabla u|^{p}\right)>N\right\}\right) \leq 10^{n s}[w]_{A_{s}}^{2} \varepsilon\left[w\left(\left\{B_{\frac{\sigma}{10}}: \mathbf{M}_{B_{1}}\left(|\nabla u|^{p}\right)>1\right\}\right)+w\left(\left\{B_{\frac{\sigma}{10}}: \mathbf{M}_{B_{1}}\left(|\mathbf{F}|^{p^{\prime}}\right)>\delta\right\}\right)\right] .
$$

Proof. Set

$$
C=\left\{B_{\frac{\sigma}{10}}: \mathrm{M}_{B_{1}}\left(|\nabla u|^{p}\right)>N\right\} \quad \text { and } \quad D=\left\{B_{\frac{\sigma}{10}}: \mathrm{M}_{B_{1}}\left(|\nabla u|^{p}\right)>1\right\} \cup\left\{B_{\frac{\sigma}{10}}: \mathrm{M}_{B_{1}}\left(|\mathbf{F}|^{p^{\prime}}\right)>\delta\right\} .
$$

Let $y$ be any point in $C$, and define

$$
m(r):=\frac{w\left(C \cap B_{\sigma r}(y)\right)}{w\left(B_{\sigma r}(y)\right)} \text { for } \quad r>0 .
$$

Due to the lower semicontinuity of $\mathrm{M}_{B_{1}}\left(|\nabla u|^{p}\right)$, we have $\lim _{r \rightarrow 0^{+}} m(r)=1$ as $C$ is open. Moreover, condition (5.6) implies that $m(r) \leq \frac{w(C)}{w\left(B \frac{\sigma}{10}\right)}<\varepsilon$ when $r \geq \frac{1}{5}$. Hence, there exists $r_{y} \in\left(0, \frac{1}{5}\right)$ such that $m\left(r_{y}\right)=\varepsilon$ and $m(r)<\varepsilon$ for all $r>r_{y}$. That is,

$$
w\left(C \cap B_{\sigma r_{y}}(y)\right)=\varepsilon w\left(B_{\sigma r_{y}}(y)\right) \quad \text { and } \quad w\left(C \cap B_{\sigma r}(y)\right)<\varepsilon w\left(B_{\sigma r}(y)\right) \quad \forall r>r_{y} .
$$

Therefore, by Vitali's covering lemma we can select a countable sequence $\left\{y_{i}\right\}_{i=1}^{\infty}$ such that $\left\{B_{\sigma r_{i}}\left(y_{i}\right)\right\}$ is a sequence of disjoint balls and

$$
C \subset \bigcup_{i=1}^{\infty} B_{5 \sigma r_{i}}\left(y_{i}\right),
$$

where $r_{i}:=r_{y_{i}}$. Since $w\left(C \cap B_{\sigma r_{i}}\left(y_{i}\right)\right)=\varepsilon w\left(B_{\sigma r_{i}}\left(y_{i}\right)\right)$ by (5.7) and $[w]_{A_{\infty}} \leq[w]_{A_{s}}$ by Lemma 2.1, it follows from Lemma5.1 that

$$
B_{\sigma r_{i}}\left(y_{i}\right) \cap B_{\frac{\sigma}{10}} \subset D .
$$

Using (5.7) - 5.8) and the first inequality in 2.17, we have

$$
\begin{aligned}
w(C) & \leq w\left(\bigcup_{i=1}^{\infty} B_{5 \sigma r_{i}}\left(y_{i}\right) \cap C\right) \leq \sum_{i=1}^{\infty} w\left(B_{5 \sigma r_{i}}\left(y_{i}\right) \cap C\right) \\
& \leq \varepsilon \sum_{i=1}^{\infty} w\left(B_{5 \sigma r_{i}}\left(y_{i}\right)\right) \leq \varepsilon[w]_{A_{s}} 5^{n s} \sum_{i=1}^{\infty} w\left(B_{\sigma r_{i}}\left(y_{i}\right)\right) .
\end{aligned}
$$


We claim that

$$
\sup _{y \in B_{\frac{\sigma}{10}}, 0<r<\frac{1}{5}} \frac{\left|B_{\sigma r}(y)\right|}{\left|B_{\sigma r}(y) \cap B_{\frac{\sigma}{10}}\right|} \leq 2^{n},
$$

which together with (2.1) gives $w\left(B_{\sigma r_{i}}\left(y_{i}\right)\right) \leq[w]_{A_{s}} 2^{n s} w\left(B_{\sigma r_{i}}\left(y_{i}\right) \cap B_{\frac{\sigma}{10}}\right)$. It follows from this and (5.9)-(5.10) that

$$
w(C) \leq \varepsilon[w]_{A_{s}}^{2} 10^{n s} \sum_{i=1}^{\infty} w\left(B_{\sigma r_{i}}\left(y_{i}\right) \cap B_{\frac{\sigma}{10}}\right)=\varepsilon[w]_{A_{s}}^{2} 10^{n s} w\left(\bigcup_{i=1}^{\infty} B_{\sigma r_{i}}\left(y_{i}\right) \cap B_{\frac{\sigma}{10}}\right) \leq \varepsilon[w]_{A_{s}}^{2} 10^{n s} w(D),
$$

which implies the desired estimate. Thus it remains to show (5.11). For this, let $y \in B_{\frac{\sigma}{10}}, r \in(0,1 / 5)$, and it is enough to consider only the case $y \neq 0$. Then the line passing through $y$ and 0 intersects $\partial B_{\sigma r}(y)$ at two distinct points, say $a_{1}$ and $a_{2}$ with $a_{1}$ being the one on the same side as the origin 0 with respect to the point $y$. If $a_{1} \notin B_{\frac{\sigma}{10}}$, then $B_{\frac{\sigma}{10}} \subset B_{\sigma r}(y)$ since for any $x \in B_{\frac{\sigma}{10}}$ we have $|x-y| \leq|x|+|y|=|x|+\left|y-a_{1}\right|-\left|a_{1}\right|<$ $\frac{\sigma}{10}+\sigma r-\frac{\sigma}{10}=\sigma r$. It follows that

$$
\frac{\left|B_{\sigma r}(y)\right|}{\left|B_{\sigma r}(y) \cap B_{\frac{\sigma}{10}}\right|}=\frac{\left|B_{\sigma r}(y)\right|}{\left|B_{\frac{\sigma}{10}}\right|}=(10 r)^{n}<2^{n} .
$$

On the other hand if $a_{1} \in B_{\frac{\sigma}{10}}$, then by letting $z$ be the midpoint of $a_{1}$ and $y$ we obviously have $B_{\frac{\sigma r}{2}}(z) \subset$ $B_{\sigma r}(y)$. If 0 belongs to the line segment $[z, y]$ connecting $z$ and $y$, then $|z|=\left|a_{1}\right|-\left|a_{1}-z\right|<\frac{\sigma}{10}-\frac{\sigma r}{2}$. In case $0 \notin[z, y]$, then $|z|=|y|-|y-z|<\frac{\sigma}{10}-\frac{\sigma r}{2}$. Thus we always have $|z|<\frac{\sigma}{10}-\frac{\sigma r}{2}$, and hence $B \frac{\sigma r}{2}(z) \subset B \frac{\sigma}{10}$ as $x \in B_{\frac{\sigma r}{2}}(z)$ implies that $|x| \leq|x-z|+|z|<\frac{\sigma r}{2}+\frac{\sigma}{10}-\frac{\sigma r}{2}=\frac{\sigma}{10}$. Therefore, we deduce that $B_{\frac{\sigma r}{2}}(z) \subset B_{\sigma r}(y) \cap B_{\frac{\sigma}{10}}$ giving

$$
\frac{\left|B_{\sigma r}(y)\right|}{\left|B_{\sigma r}(y) \cap B_{\frac{\sigma}{10}}\right|} \leq \frac{\left|B_{\sigma r}(y)\right|}{\left|B_{\frac{\sigma r}{2}}(z)\right|}=2^{n} .
$$

We then conclude that (5.11) holds, and the proof is complete.

\subsection{Gradient estimates in weighted $L^{q}$ spaces}

We are now ready to prove Theorem 2.4

Proof of Theorem 2.4 Let $N=N(p, n, \Lambda)>1$ be as in Lemma 5.2, and let $l=q / p \geq 1$. We choose $\varepsilon=\varepsilon\left(p, q, n, \Lambda, s,[w]_{A_{s}}\right)>0$ be such that

$$
\varepsilon_{1} \stackrel{\text { def }}{=} 10^{n s}[w]_{A_{s}}^{2} \varepsilon=\frac{1}{2 N^{l}},
$$

and let $\delta$ and $\sigma$ (depending only on $p, q, n, \omega, \Lambda, M_{0}, s$, and $[w]_{A_{s}}$ ) be the corresponding positive constants given by Lemma5.2. Assuming for a moment that $u$ satisfies

$$
w\left(\left\{B_{\frac{\sigma}{10}}: \mathrm{M}_{B_{1}}\left(|\nabla u|^{p}\right)>N\right\}\right)<\varepsilon w\left(B_{\frac{\sigma}{10}}\right) .
$$

Then it follows from Lemma 5.2 that

$$
w\left(\left\{B_{\frac{\sigma}{10}}: \mathbf{M}_{B_{1}}\left(|\nabla u|^{p}\right)>N\right\}\right) \leq \varepsilon_{1}\left[w\left(\left\{B_{\frac{\sigma}{10}}: \mathbf{M}_{B_{1}}\left(|\nabla u|^{p}\right)>1\right\}\right)+w\left(\left\{B_{\frac{\sigma}{10}}: \mathbf{M}_{B_{1}}\left(|\mathbf{F}|^{p^{\prime}}\right)>\delta\right\}\right)\right] .
$$


Let us iterate this estimate by considering

$$
u_{1}(x)=\frac{u(x)}{N^{\frac{1}{p}}}, \quad \mathbf{F}_{1}(x)=\frac{\mathbf{F}(x)}{N^{\frac{p-1}{p}}} \quad \text { and } \quad \lambda_{1}=N^{\frac{1}{p}} \lambda .
$$

It is clear that $\left\|u_{1}\right\|_{L^{\infty}\left(B_{1}\right)} \leq \frac{M_{0}}{\lambda_{1} \theta}$ and $u_{1}$ is a weak solution of $\operatorname{div}\left[\frac{\mathbf{A}\left(x, \lambda_{1} \theta u_{1}, \lambda_{1} \nabla u_{1}\right)}{\lambda_{1}^{p-1}}\right]=\operatorname{div} \mathbf{F}_{1}$ in $B_{10}$. Moreover, thanks to (5.12) we have

$$
w\left(\left\{B_{\frac{\sigma}{10}}: \mathrm{M}_{B_{1}}\left(\left|\nabla u_{1}\right|^{p}\right)>N\right\}\right)=w\left(\left\{B_{\frac{\sigma}{10}}: \mathrm{M}_{B_{1}}\left(|\nabla u|^{p}\right)>N^{2}\right\}\right)<\varepsilon w\left(B_{\frac{\sigma}{10}}\right) .
$$

Therefore, by applying Lemma 5.2 to $u_{1}$ we obtain

$$
\begin{aligned}
w\left(\left\{B_{\frac{\sigma}{10}}: \mathbf{M}_{B_{1}}\left(\left|\nabla u_{1}\right|^{p}\right)>N\right\}\right) & \leq \varepsilon_{1}\left[w\left(\left\{B_{\frac{\sigma}{10}}: \mathbf{M}_{B_{1}}\left(\left|\nabla u_{1}\right|^{p}\right)>1\right\}\right)+w\left(\left\{B_{\frac{\sigma}{10}}: \mathbf{M}_{B_{1}}\left(\left|\mathbf{F}_{1}\right|^{p^{\prime}}\right)>\delta\right\}\right)\right] \\
& =\varepsilon_{1}\left[w\left(\left\{B_{\frac{\sigma}{10}}: \mathbf{M}_{B_{1}}\left(|\nabla u|^{p}\right)>N\right\}\right)+w\left(\left\{B_{\frac{\sigma}{10}}: \mathbf{M}_{B_{1}}\left(|\mathbf{F}|^{p^{\prime}}\right)>\delta N\right\}\right)\right] .
\end{aligned}
$$

We infer from this and (5.13) that

$$
\begin{aligned}
& w\left(\left\{B_{\frac{\sigma}{10}}: \mathbf{M}_{B_{1}}\left(|\nabla u|^{p}\right)>N^{2}\right\}\right) \leq \varepsilon_{1}^{2} w\left(\left\{B_{\frac{\sigma}{10}}: \mathbf{M}_{B_{1}}\left(|\nabla u|^{p}\right)>1\right\}\right) \\
& \quad+\varepsilon_{1}^{2} w\left(\left\{B_{\frac{\sigma}{10}}: \mathbf{M}_{B_{1}}\left(|\mathbf{F}|^{p^{\prime}}\right)>\delta\right\}\right)+\varepsilon_{1} w\left(\left\{B_{\frac{\sigma}{10}}: \mathbf{M}_{B_{1}}\left(|\mathbf{F}|^{p^{\prime}}\right)>\delta N\right\}\right) .
\end{aligned}
$$

Next, let

$$
u_{2}(x)=\frac{u(x)}{N^{\frac{2}{p}}}, \quad \mathbf{F}_{2}(x)=\frac{\mathbf{F}(x)}{N^{\frac{2(p-1)}{p}}} \quad \text { and } \quad \lambda_{2}=N^{\frac{2}{p}} \lambda .
$$

Then $u_{2}$ is a weak solution of $\operatorname{div}\left[\frac{\mathbf{A}\left(x, \lambda_{2} \theta u_{2}, \lambda_{2} \nabla u_{2}\right)}{\lambda_{2}^{p-1}}\right]=\operatorname{div} \mathbf{F}_{2}$ in $B_{10}$ satisfying $\left\|u_{2}\right\|_{L^{\infty}\left(B_{1}\right)} \leq M_{0} /\left(\lambda_{2} \theta\right)$ and

$$
w\left(\left\{B_{\frac{\sigma}{10}}: \mathrm{M}_{B_{1}}\left(\left|\nabla u_{2}\right|^{p}\right)>N\right\}\right)=w\left(\left\{B_{\frac{\sigma}{10}}: \mathrm{M}_{B_{1}}\left(|\nabla u|^{p}\right)>N^{3}\right\}\right)<\varepsilon w\left(B_{\frac{\sigma}{10}}\right) .
$$

Hence by applying Lemma 5.2 to $u_{2}$ we get

$$
\begin{aligned}
w\left(\left\{B_{\frac{\sigma}{10}}: \mathbf{M}_{B_{1}}\left(\left|\nabla u_{2}\right|^{p}\right)>N\right\}\right) & \leq \varepsilon_{1}\left[w\left(\left\{B_{\frac{\sigma}{10}}: \mathbf{M}_{B_{1}}\left(\left|\nabla u_{2}\right|^{p}\right)>1\right\}\right)+w\left(\left\{B_{\frac{\sigma}{10}}: \mathbf{M}_{B_{1}}\left(\left|\mathbf{F}_{2}\right|^{p^{\prime}}\right)>\delta\right\}\right)\right] \\
& =\varepsilon_{1}\left[w\left(\left\{B_{\frac{\sigma}{10}}: \mathbf{M}_{B_{1}}\left(|\nabla u|^{p}\right)>N^{2}\right\}\right)+w\left(\left\{B_{\frac{\sigma}{10}}: \mathbf{M}_{B_{1}}\left(|\mathbf{F}|^{p^{\prime}}\right)>\delta N^{2}\right\}\right)\right] .
\end{aligned}
$$

This together with 5.14 gives

$$
w\left(\left\{B_{\frac{\sigma}{10}}: \mathbf{M}_{B_{1}}\left(|\nabla u|^{p}\right)>N^{3}\right\}\right) \leq \varepsilon_{1}^{3} w\left(\left\{B_{\frac{\sigma}{10}}: \mathbf{M}_{B_{1}}\left(|\nabla u|^{p}\right)>1\right\}\right)+\sum_{i=1}^{3} \varepsilon_{1}^{i} w\left(\left\{B_{\frac{\sigma}{10}}: \mathbf{M}_{B_{1}}\left(|\mathbf{F}|^{p^{\prime}}\right)>\delta N^{3-i}\right\}\right) .
$$

By repeating the iteration, we then conclude that

$$
w\left(\left\{B_{\frac{\sigma}{10}}: \mathbf{M}_{B_{1}}\left(|\nabla u|^{p}\right)>N^{k}\right\}\right) \leq \varepsilon_{1}^{k} w\left(\left\{B_{\frac{\sigma}{10}}: \mathbf{M}_{B_{1}}\left(|\nabla u|^{p}\right)>1\right\}\right)+\sum_{i=1}^{k} \varepsilon_{1}^{i} w\left(\left\{B_{\frac{\sigma}{10}}: \mathbf{M}_{B_{1}}\left(|\mathbf{F}|^{p^{\prime}}\right)>\delta N^{k-i}\right\}\right)
$$


for all $k=1,2, \ldots$ This together with

$$
\begin{aligned}
& \int_{B_{\frac{\sigma}{10}}} \mathrm{M}_{B_{1}}\left(|\nabla u|^{p}\right)^{l} d w=l \int_{0}^{\infty} t^{l-1} w\left(\left\{B_{\frac{\sigma}{10}}: \mathrm{M}_{B_{1}}\left(|\nabla u|^{p}\right)>t\right\}\right) d t \\
& =l \int_{0}^{N} t^{l-1} w\left(\left\{B_{\frac{\sigma}{10}}: \mathrm{M}_{B_{1}}\left(|\nabla u|^{p}\right)>t\right\}\right) d t+l \sum_{k=1}^{\infty} \int_{N^{k}}^{N^{k+1}} t^{l-1} w\left(\left\{B_{\frac{\sigma}{10}}: \mathrm{M}_{B_{1}}\left(|\nabla u|^{p}\right)>t\right\}\right) d t \\
& \leq N^{l} w\left(B_{\frac{\sigma}{10}}\right)+\left(N^{l}-1\right) \sum_{k=1}^{\infty} N^{l k} w\left(\left\{B_{\frac{\sigma}{10}}: \mathrm{M}_{B_{1}}\left(|\nabla u|^{p}\right)>N^{k}\right\}\right)
\end{aligned}
$$

gives

$$
\begin{aligned}
\int_{B_{\frac{\sigma}{10}}} \mathrm{M}_{B_{1}}\left(|\nabla u|^{p}\right)^{l} d w \leq & N^{l} w\left(B_{\frac{\sigma}{10}}\right)+\left(N^{l}-1\right) w\left(B_{\frac{\sigma}{10}}\right) \sum_{k=1}^{\infty}\left(\varepsilon_{1} N^{l}\right)^{k} \\
& +\sum_{k=1}^{\infty} \sum_{i=1}^{k}\left(N^{l}-1\right) N^{l k} \varepsilon_{1}^{i} w\left(\left\{B_{\frac{\sigma}{10}}: \mathbf{M}_{B_{1}}\left(|\mathbf{F}|^{p^{\prime}}\right)>\delta N^{k-i}\right\}\right) .
\end{aligned}
$$

But we have

$$
\begin{aligned}
& \sum_{k=1}^{\infty} \sum_{i=1}^{k}\left(N^{l}-1\right) N^{l k} \varepsilon_{1}^{i} w\left(\left\{B_{\frac{\sigma}{10}}: \mathrm{M}_{B_{1}}\left(|\mathbf{F}|^{p^{\prime}}\right)>\delta N^{k-i}\right\}\right) \\
& =\left(\frac{N}{\delta}\right)^{l} \sum_{i=1}^{\infty}\left(\varepsilon_{1} N^{l}\right)^{i}\left[\sum_{k=i}^{\infty}\left(N^{l}-1\right) \delta^{l} N^{l(k-i-1)} w\left(\left\{B_{\frac{\sigma}{10}}: \mathrm{M}_{B_{1}}\left(|\mathbf{F}|^{p^{\prime}}\right)>\delta N^{k-i}\right\}\right)\right] \\
& =\left(\frac{N}{\delta}\right)^{l} \sum_{i=1}^{\infty}\left(\varepsilon_{1} N^{l}\right)^{i}\left[\sum_{j=0}^{\infty}\left(N^{l}-1\right) \delta^{l} N^{l(j-1)} w\left(\left\{B_{\frac{\sigma}{10}}: \mathrm{M}_{B_{1}}\left(|\mathbf{F}|^{p^{\prime}}\right)>\delta N^{j}\right\}\right)\right] \\
& \leq\left(\frac{N}{\delta}\right)^{l}\left[\int_{B_{\frac{\sigma}{10}}} \mathrm{M}_{B_{1}}\left(|\mathbf{F}|^{p^{\prime}}\right)^{l} d w\right] \sum_{i=1}^{\infty}\left(\varepsilon_{1} N^{l}\right)^{i} .
\end{aligned}
$$

Thus we infer that

$$
\begin{aligned}
\int_{B_{\frac{\sigma}{10}}} \mathbf{M}_{B_{1}}\left(|\nabla u|^{p}\right)^{l} d w & \leq N^{l} w\left(B_{\frac{\sigma}{10}}\right)+\left[\left(N^{l}-1\right) w\left(B_{\frac{\sigma}{10}}\right)+\left(\frac{N}{\delta}\right)^{l} \int_{B_{\frac{\sigma}{10}}} \mathbf{M}_{B_{1}}\left(|\mathbf{F}|^{p^{\prime}}\right)^{l} d w\right] \sum_{k=1}^{\infty}\left(\varepsilon_{1} N^{l}\right)^{k} \\
& =N^{l} w\left(B_{\frac{\sigma}{10}}\right)+\left[\left(N^{l}-1\right) w\left(B_{\frac{\sigma}{10}}\right)+\left(\frac{N}{\delta}\right)^{l} \int_{B_{\frac{\sigma}{10}}} \mathbf{M}_{B_{1}}\left(|\mathbf{F}|^{p^{\prime}}\right)^{l} d w\right] \sum_{k=1}^{\infty} 2^{-k} \\
& \leq C\left(w\left(B_{\frac{\sigma}{10}}\right)+\int_{B_{\frac{\sigma}{10}}} \mathrm{M}_{B_{1}}\left(|\mathbf{F}|^{p^{\prime}}\right)^{l} d w\right)
\end{aligned}
$$


with the constant $C$ depending only on $p, q, n, \omega, \Lambda, M_{0}, s$, and $[w]_{A_{s}}$. This together with the facts $l=q / p$ and $|\nabla u(x)|^{p} \leq \mathrm{M}_{B_{1}}\left(|\nabla u|^{p}\right)(x)$ for a.e. $x \in B_{\frac{\sigma}{10}}$ yields

$$
f_{B_{\frac{\sigma}{10}}}|\nabla u|^{q} d w \leq C\left(1+f_{B_{\frac{\sigma}{10}}} \mathbf{M}_{B_{1}}\left(|\mathbf{F}|^{p^{\prime}}\right)^{\frac{q}{p}} d w\right) .
$$

We next remove the extra assumption (5.12) for $u$. Notice that for any $M>0$, by using the weak type $1-1$ estimate for the maximal function we get

$$
\left|\left\{B_{\frac{\sigma}{10}}: \mathrm{M}_{B_{1}}\left(|\nabla u|^{p}\right)>N M^{p}\right\}\right| \leq \frac{C}{N M^{p}} \int_{B_{1}}|\nabla u|^{p} d x .
$$

Let $\bar{u}(x, t)=u(x, t) / M$, where

$$
M^{p}:=\frac{2 C\|\nabla u\|_{L^{p}\left(B_{1}\right)}^{p}}{N\left(\varepsilon K^{-1}\right)^{\frac{1}{\beta}}\left|B_{\frac{\sigma}{10}}\right|}
$$

with $K$ and $\beta$ being the constants given by $(2.1)$ and depending only on $n$ and $[w]_{A_{s}}$. Then it follows from (5.16) that

$$
\left|\left\{B_{\frac{\sigma}{10}}: \mathrm{M}_{B_{1}}\left(|\nabla \bar{u}|^{p}\right)>N\right\}\right| \leq \frac{1}{2}\left(\varepsilon K^{-1}\right)^{\frac{1}{\beta}}\left|B_{\frac{\sigma}{10}}\right| .
$$

This together with the second inequality in (2.1) gives

$$
w\left(\left\{B_{\frac{\sigma}{10}}: \mathrm{M}_{B_{1}}\left(|\nabla \bar{u}|^{p}\right)>N\right\}\right)<\varepsilon w\left(B_{\frac{\sigma}{10}}\right) .
$$

Hence we can apply (5.15) to $\bar{u}$ with $\mathbf{F}$ and $\lambda$ being replaced by $\overline{\mathbf{F}}=\mathbf{F} / M^{p-1}$ and $\bar{\lambda}=\lambda M$. By reversing back to the functions $u$ and $\mathbf{F}$, we then obtain

$$
f_{B_{\frac{\sigma}{10}}}|\nabla u|^{q} d w \leq C\left(M^{q}+f_{B_{\frac{\sigma}{10}}} \mathbf{M}_{B_{1}}\left(|\mathbf{F}|^{p^{\prime}}\right)^{\frac{q}{p}} d w\right) \leq C\left(\|\nabla u\|_{L^{p}\left(B_{1}\right)}^{q}+f_{B_{\frac{\sigma}{10}}} \mathbf{M}_{B_{1}}\left(|\mathbf{F}|^{p^{\prime}}\right)^{\frac{q}{p}} d w\right) .
$$

Due to the translation invariance of our equation, the above estimate holds true if $B_{\frac{\sigma}{10}}$ and $B_{1}$ are respectively replaced by $B_{\frac{\sigma}{10}}(z) \subset B_{\frac{3}{2}}$ and $B_{1}(z) \subset B_{2}$ for any $z \in B_{1}$. Therefore, desired estimate (2.7) follows by covering $B_{1}$ by a finite number of balls $B_{\frac{\sigma}{10}}(z)$ with $z \in B_{1}$.

\subsection{Gradient estimates in weighted Morrey spaces}

Proof of Theorem 1.2 Let $\bar{x} \in B_{1}$ and $0<r \leq 2$. Then by rescaling and applying Theorem 2.4 we obtain

$$
f_{B_{r}(\bar{x})}|\nabla u|^{q} d w \leq C\left[\left(f_{B_{2 r}(\bar{x})}|\nabla u|^{p} d x\right)^{\frac{q}{p}}+f_{B_{\frac{3}{2} r}(\bar{x})} \mathrm{M}_{B_{2 r}(\bar{x})}\left(|\mathbf{F}|^{p^{\prime}}\right)^{\frac{q}{p}} d w\right] .
$$

Since $B_{\frac{3}{2} r}(\bar{x}) \subset B_{4}$ and $B_{2 r}(\bar{x}) \subset B_{5}$, it then follows that

$$
\varphi\left(B_{r}(\bar{x})\right) f_{B_{r}(\bar{x})}|\nabla u|^{q} d w \leq C\left[\varphi\left(B_{r}(\bar{x})\right)\left(f_{B_{2 r}(\bar{x})}|\nabla u|^{p} d x\right)^{\frac{q}{p}}+\frac{\varphi\left(B_{r}(\bar{x})\right)}{w\left(B_{\frac{3}{2} r}(\bar{x})\right)} \int_{B_{\frac{3}{2} r}(\bar{x}) \cap B_{4}} \mathrm{M}_{B_{5}}\left(|\mathbf{F}|^{p^{\prime}}\right)^{\frac{q}{p}} d w\right]
$$


We next estimate the first term in the above right hand side. For this, let $\varepsilon \in(0, n)$ to be determined later and use the trick in [25, Page 2506] to write

$$
f_{B_{2 r}(\bar{x})}|\nabla u|^{p} d x=\omega_{n}^{-1}(2 r)^{-\varepsilon} \int_{B_{2 r}(\bar{x})}|\nabla u|^{p} \bar{w} d x \leq \omega_{n}^{-1}(2 r)^{-\varepsilon} \int_{B_{5}}|\nabla u|^{p} \bar{w} d x
$$

with $\omega_{n}:=\left|B_{1}\right|$ and $\bar{w}$ being the weight defined by

$$
\bar{w}(x):=\min \left\{|x-\bar{x}|^{-n+\varepsilon},(2 r)^{-n+\varepsilon}\right\} .
$$

As $\bar{w} \in A_{t}$ with $[\bar{w}]_{A_{t}} \leq C(t, \varepsilon, n)$ for any $1<t<\infty$ (see [25, Lemma 3.2]), we can apply Theorem 2.4 with $q=p$ to estimate the above last integral. As a consequence, we obtain

$$
\begin{aligned}
f_{B_{2 r}(\bar{x})}|\nabla u|^{p} d x & \leq C(2 r)^{-\varepsilon}\left(\bar{w}\left(B_{5}\right)\|\nabla u\|_{L^{p}\left(B_{10}\right)}^{p}+\int_{B_{\frac{15}{2}}} \mathrm{M}_{B_{10}}\left(|\mathbf{F}|^{p^{\prime}}\right) \bar{w} d x\right) \\
& \leq C(2 r)^{-\varepsilon}\left(\|\nabla u\|_{L^{p}\left(B_{10}\right)}^{p}+\int_{B_{\frac{15}{2}}} \mathrm{M}_{B_{10}}\left(|\mathbf{F}|^{p^{\prime}}\right) \bar{w} d x\right)
\end{aligned}
$$

with $C>0$ depending only on $p, n, \omega, \Lambda, M_{0}$, and $\varepsilon$. Notice that to obtain the last inequality we have used the fact

$$
\bar{w}\left(B_{5}\right) \leq \int_{B_{6}(\bar{x})}|x-\bar{x}|^{-n+\varepsilon} d x=\omega_{n} \int_{0}^{6} t^{\varepsilon-1} d t=\frac{\omega_{n}}{\varepsilon} 6^{\varepsilon} .
$$

To bound the last integral in (5.18), we employ Fubini's theorem to get

$$
\begin{aligned}
\int_{B_{\frac{15}{2}}} \mathbf{M}_{B_{10}}\left(|\mathbf{F}|^{p^{\prime}}\right) \bar{w} d x & =\int_{0}^{\infty} \int_{\left\{B_{\frac{15}{2}}: \bar{w}(x)>t\right\}} \mathbf{M}_{B_{10}}\left(|\mathbf{F}|^{p^{\prime}}\right) d x d t \leq \int_{0}^{(2 r)^{-n+\varepsilon}} \int_{B_{t^{(-n+\varepsilon)^{-1}}(\bar{x}) \cap B_{\frac{15}{2}}}} \mathbf{M}_{B_{10}}\left(|\mathbf{F}|^{p^{\prime}}\right) d x d t \\
& \leq \int_{0}^{15^{-n+\varepsilon}} \int_{B_{\frac{15}{2}}} \mathbf{M}_{B_{10}}\left(|\mathbf{F}|^{p^{\prime}}\right) d x d t+\int_{15^{-n+\varepsilon}}^{(2 r)^{-n+\varepsilon}} \int_{B_{t^{(-n+\varepsilon)^{-1}}(\bar{x}) \cap B_{\frac{15}{2}}}} \mathbf{M}_{B_{10}}\left(|\mathbf{F}|^{p^{\prime}}\right) d x d t .
\end{aligned}
$$

Since $B_{\frac{15}{2}} \subset B_{\frac{17}{2}}(\bar{x})$, we have $B_{\frac{15}{2}}=B_{\frac{17}{2}}(\bar{x}) \cap B_{\frac{15}{2}}$ and then deduce that

$$
\int_{B_{\frac{15}{2}}} \mathbf{M}_{B_{10}}\left(|\mathbf{F}|^{p^{\prime}}\right) \bar{w} d x \leq C\left\|\mathbf{M}_{B_{10}}\left(|\mathbf{F}|^{p^{\prime}}\right)\right\|_{\mathcal{M}^{1, \varphi^{4}}\left(B_{\frac{15}{2}}\right)}\left[\varphi\left(B_{\frac{17}{2}}(\bar{x})\right)^{\frac{-p}{q}}+\int_{15^{-n+\varepsilon}}^{(2 r)^{-n+\varepsilon}} t^{\frac{n}{-n+\varepsilon}} \varphi\left(B_{t^{(-n+\varepsilon)^{-1}}}(\bar{x})\right)^{\frac{-p}{q}} d t\right],
$$

where we recall that $\mathcal{M}^{1, \varphi}(U)$ denotes the Morrey space $\mathcal{M}_{w}^{1, \varphi}(U)$ with $w=1$. As $\varphi \in \mathcal{B}_{+}$by the assumption and $\left\{\mathcal{B}_{\alpha}\right\}$ is decreasing in $\alpha$, there exists $\alpha \in(0, n)$ such that $\varphi \in \mathcal{B}_{\alpha}$. It then follows if $\varepsilon<\alpha p / q$ that

$$
\begin{aligned}
\int_{B_{\frac{15}{2}}} \mathrm{M}_{B_{10}}\left(|\mathbf{F}|^{p^{\prime}}\right) \bar{w} d x & \leq C(2 r)^{\alpha \frac{p}{q}} \varphi\left(B_{2 r}(\bar{x})\right)^{\frac{-p}{q}}\left\|\mathbf{M}_{B_{10}}\left(|\mathbf{F}|^{p^{\prime}}\right)\right\|_{\mathcal{M}^{1, \varphi}{ }^{\frac{p}{q}}\left(B_{\frac{15}{2}}\right.}\left[1+\int_{0}^{(2 r)^{-n+\varepsilon}} t^{\frac{n-\alpha+\frac{p}{q}}{-n+\varepsilon}} d t\right] \\
& \leq C(2 r)^{\varepsilon} \varphi\left(B_{r}(\bar{x})\right)^{\frac{-p}{q}}\left\|\mathbf{M}_{B_{10}}\left(|\mathbf{F}|^{p^{\prime}}\right)\right\|_{\mathcal{M}^{1, \varphi^{\frac{p}{q}}}\left(B_{\frac{15}{2}}\right)} .
\end{aligned}
$$


Combining this with (5.18), we arrive at:

$$
f_{B_{2 r}(\bar{x})}|\nabla u|^{p} d x \leq C\left(r^{-\varepsilon}\|\nabla u\|_{L^{p}\left(B_{10}\right)}^{p}+\varphi\left(B_{r}(\bar{x})\right)^{\frac{-p}{q}}\left\|\mathbf{M}_{B_{10}}\left(|\mathbf{F}|^{p^{\prime}}\right)\right\|_{\mathcal{M}^{1, \varphi^{q}}\left(B_{\frac{15}{2}}\right.}\right) .
$$

Therefore, we infer from (5.17) and the fact $\varphi \in \mathcal{B}_{\alpha}$ that

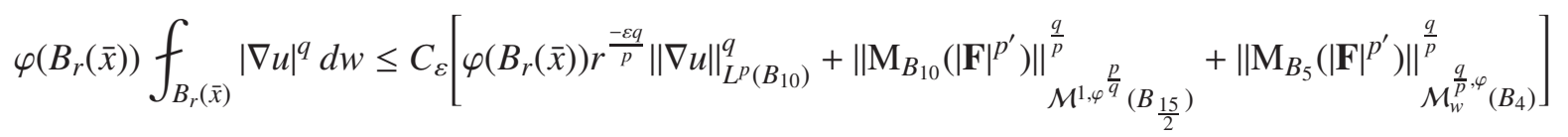

$$
\begin{aligned}
& \leq C_{\varepsilon}\left[\varphi\left(B_{2}(\bar{x})\right) r^{\alpha-\frac{\varepsilon q}{p}}\|\nabla u\|_{L^{p}\left(B_{10}\right)}^{q}+\left\|\mathbf{M}_{B_{10}}\left(|\mathbf{F}|^{p^{\prime}}\right)\right\|_{\mathcal{M}^{1, \varphi} \frac{q}{q}\left(B_{\frac{15}{2}}\right)}^{\frac{q}{p}}+\left\|\mathbf{M}_{B_{5}}\left(|\mathbf{F}|^{p^{\prime}}\right)\right\|_{\mathcal{M}_{w}^{\frac{q}{p}}}^{\frac{q}{p},\left(B_{4}\right)}\right]
\end{aligned}
$$

for all $\bar{x} \in B_{1}$ and $0<r \leq 2$. By taking $\varepsilon=\frac{\alpha}{2} \frac{p}{q}$ and as $\sup _{\bar{x} \in B_{1}} \varphi\left(B_{2}(\bar{x})\right)<\infty$, this gives estimate (1.9).

Proof of Theorem 1.3 Since $v \in A_{\frac{q}{p}}$, Lemma2.1 gives $v^{1-\left(\frac{q}{p}\right)^{\prime}} \in A_{\left(\frac{q}{p}\right)^{\prime}} \subset A_{\infty}$. Thus our assumptions imply that condition (B) in Corollary 3.6 is satisfied. Also as $\varphi \in \mathcal{B}_{0}$, it is clear that (1.11) implies (3.6). Indeed, for any $y \in \mathbb{R}^{n}$ and any $s \geq 2 r>0$ we have from (1.11) and $\varphi \in \mathcal{B}_{0}$ that

$$
\frac{v\left(B_{s}(y)\right)}{w\left(B_{s}(y)\right)} \frac{1}{\phi\left(B_{s}(y)\right)} \leq C_{*} \frac{1}{\varphi\left(B_{\frac{s}{2}}(y)\right)} \leq C_{*} C \frac{1}{\varphi\left(B_{r}(y)\right)}
$$

yielding (3.6). Moreover, by [14, Theorem 9.3.3] there exist $s \in(1, \infty)$ and $C>0$ depending only on $n$ and $[w]_{A_{\infty}}$ such that $[w]_{A_{s}} \leq C$. Therefore, it follows from Theorem 1.2 and Corollary 3.6 that

$$
\|\nabla u\|_{\mathcal{M}_{w}^{q, \varphi}\left(B_{1}\right)} \leq C\left(\|\nabla u\|_{L^{p}\left(B_{10}\right)}+\left\|\mathbf{M}_{B_{10}}\left(|\mathbf{F}|^{p^{\prime}}\right)\right\|_{\mathcal{M}^{1, \varphi}{ }^{\frac{p}{q}}\left(B_{10}\right)}^{\frac{1}{p}}+\left\||\mathbf{F}|^{\frac{1}{p-1}}\right\|_{\mathcal{M}_{v}^{q, \phi}\left(B_{10}\right)}\right)
$$

with $C>0$ depending only on $q, p, n, \omega, \Lambda, M_{0}, \varphi, C_{*},[w]_{A_{\infty}},[v]_{A_{\frac{q}{p}}}$, and $\left[w, v^{1-\left(\frac{q}{p}\right)^{\prime}}\right]_{A_{\frac{q}{p}}}$. Thus it remains to estimate the middle term on the right hand side of (5.19). Let $l:=q / p>1$. Then for any nonnegative function $g \in L^{1}\left(B_{10}\right)$, we obtain from Hölder inequality and assumption (1.10) that

$$
\begin{aligned}
\frac{\varphi\left(B_{R}(\bar{x})\right)}{\left|B_{R}(\bar{x})\right|^{l}}\left(\int_{B_{R}(\bar{x}) \cap B_{10}} g d x\right)^{l} & \leq \frac{\varphi\left(B_{R}(\bar{x})\right)}{\left|B_{R}(\bar{x})\right|^{l}}\left(\int_{B_{R}(\bar{x}) \cap B_{10}} g^{l} v d x\right)\left(\int_{B_{R}(\bar{x})} v^{1-l^{\prime}}\right)^{l-1} \\
& \leq\left[w, v^{1-l^{\prime}}\right]_{A_{l}} \frac{\varphi\left(B_{R}(\bar{x})\right)}{w\left(B_{R}(\bar{x})\right)} \int_{B_{R}(\bar{x}) \cap B_{10}} g^{l} v d x \leq\left[w, v^{1-l^{\prime}}\right]_{A_{l}}\|g\|_{\mathcal{M}_{v}^{l, \hat{\varphi}}\left(B_{10}\right)}^{l}
\end{aligned}
$$

for all $\bar{x} \in B_{10}$ and all $0<R \leq 20$, where

$$
\hat{\varphi}(B):=\frac{v(B)}{w(B)} \varphi(B)
$$

Hence we infer that

$$
\left\|\mathbf{M}_{B_{10}}\left(|\mathbf{F}|^{p^{\prime}}\right)\right\|_{\mathcal{M}^{1, \varphi^{\top}}\left(B_{10}\right)} \leq\left[w, v^{1-l^{\prime}}\right]_{A_{l}}^{\frac{1}{l}}\left\|\mathbf{M}_{B_{10}}\left(|\mathbf{F}|^{p^{\prime}}\right)\right\|_{\mathcal{M}_{v}^{l, \hat{\varphi}}\left(B_{10}\right)} .
$$


Using $\phi \in \mathcal{B}_{0}$, condition (1.11), and the doubling property of $w$ due to Lemma 2.1, we have

$$
\sup _{s \geq 2 r} \frac{1}{\phi\left(B_{s}(y)\right)} \leq C \frac{1}{\phi\left(B_{2 r}(y)\right)} \leq C C_{*} \frac{w\left(B_{2 r}(y)\right)}{v\left(B_{2 r}(y)\right)} \frac{1}{\varphi\left(B_{r}(y)\right)} \leq C^{\prime} \frac{w\left(B_{r}(y)\right)}{v\left(B_{r}(y)\right)} \frac{1}{\varphi\left(B_{r}(y)\right)}=C^{\prime} \frac{1}{\hat{\varphi}\left(B_{r}(y)\right)}
$$

for all $y \in \mathbb{R}^{n}$ and $r>0$. Thus as $v \in A_{l}$ we can use the strong type estimate for the Hardy-Littlewood maximal function given by Corollary 3.7 to estimate the right hand side of (5.20). As a result, we get

$$
\left\|\mathrm{M}_{B_{10}}\left(|\mathbf{F}|^{p^{\prime}}\right)\right\|_{\mathcal{M}^{1, \varphi^{1}}\left(B_{10}\right)} \leq C\left|\left\|\left.\mathbf{F}\right|^{p^{\prime}}\right\|_{\mathcal{M}_{v}^{l, \phi}\left(B_{10}\right)}=C\|\| \mathbf{F}\right|^{\frac{1}{p-1}} \|_{\mathcal{M}_{v}^{q, \phi}\left(B_{10}\right)}^{p} .
$$

This and (5.19) yield desired estimate (1.12).

We close the paper by noting that if $w \in A_{\frac{q}{p}}$, then the assumption $v \in A_{\frac{q}{p}}$ in Theorem 1.3 can be disposed if condition 1.10 is strengthened by assuming that there exists $r>1$ such that

$$
\sup _{B}\left(f_{B} w d x\right)\left(f_{B} v^{r\left[1-\left(\frac{q}{p}\right)^{\prime}\right]} d x\right)^{\frac{q-p}{p r}}<\infty .
$$

This follows from the above proof of Theorem 1.3 and the fact that condition (A) in Corollary 3.6 is satisfied in this case. Indeed, having (A) allows us to use Corollary 3.6 to obtain (5.19) and it remains to show (5.21). But for any nonnegative function $g \in L^{1}\left(B_{10}\right)$, we have from Hölder inequality and $w \in A_{l}$ that

$$
\begin{aligned}
\frac{\varphi\left(B_{R}(\bar{x})\right)}{\left|B_{R}(\bar{x})\right|^{l}}\left(\int_{B_{R}(\bar{x}) \cap B_{10}} g d x\right)^{l} & \leq \frac{\varphi\left(B_{R}(\bar{x})\right)}{\left|B_{R}(\bar{x})\right|^{l}}\left(\int_{B_{R}(\bar{x}) \cap B_{10}} g^{l} w d x\right)\left(\int_{B_{R}(\bar{x})} w^{1-l^{\prime}}\right)^{l-1} \\
& \leq[w]_{A_{l}} \frac{\varphi\left(B_{R}(\bar{x})\right)}{w\left(B_{R}(\bar{x})\right)} \int_{B_{R}(\bar{x}) \cap B_{10}} g^{l} w d x \leq[w]_{A_{l}}\|g\|_{\mathcal{M}_{w}^{l, \varphi}\left(B_{10}\right)}^{l}
\end{aligned}
$$

for all $\bar{x} \in B_{10}$ and all $0<R \leq 20$. This observation together with Corollary 3.6 gives

$$
\left\|\mathbf{M}_{B_{10}}\left(|\mathbf{F}|^{p^{\prime}}\right)\right\|_{\mathcal{M}^{1, \varphi}{ }^{\frac{1}{T}}\left(B_{10}\right)} \leq[w]_{A_{l}}^{\frac{1}{l}}\left\|\mathbf{M}_{B_{10}}\left(|\mathbf{F}|^{p^{\prime}}\right)\right\|_{\mathcal{M}_{w}^{l, \varphi}\left(B_{10}\right)} \leq\left. C\|\| \mathbf{F}\right|^{p^{\prime}}\left\|_{\mathcal{M}_{v}^{l, \phi}\left(B_{10}\right)}=C\right\|\left\|\left.\mathbf{F}\right|^{\frac{1}{p-1}}\right\|_{\mathcal{M}_{v}^{q, \phi}\left(B_{10}\right)}^{p}
$$

which is precisely (5.21). As a consequence, we still arrive at conclusion (1.12).

Acknowledgement The authors would like to thank the anonymous referee for valuable suggestions which improve the presentation of the paper.

\section{References}

[1] E. Acerbi and G. Mingione. Gradient estimates for a class of parabolic systems. Duke Math. J. 136 (2007), no. 2, 285-320.

[2] S-S. Byun, D. Palagachev and P. Shin. Global Sobolev regularity for general elliptic equations of p-Laplacian type. Calc. Var. Partial Differential Equations 57 (2018), no. 5, Art. 135, 19 pp. 
[3] S-S. Byun and S. Ryu. Global weighted estimates for the gradient of solutions to nonlinear elliptic equations. Ann. Inst. H. Poincar Anal. Non Linaire 30 (2013), no. 2, 291-313.

[4] L. Caffarelli and I. Peral. On $W^{1, p}$ estimates for elliptic equations in divergence form. Comm. Pure Appl. Math. 51 (1998), no. 1, 1-21.

[5] F. Chiarenza and M. Frasca. Morrey spaces and Hardy-Littlewood maximal function. Rend. Mat. Appl. 7 (1987), no. 3-4, 273-279.

[6] E. DiBenedetto. $C^{1+\alpha}$ local regularity of weak solutions of degenerate elliptic equations. Nonlinear Anal. 7 (1983), no. 8, 827-850.

[7] E. DiBenedetto and J. Manfredi. On the higher integrability of the gradient of weak solutions of certain degenerate elliptic systems. Amer. J. Math. 115 (1993), no. 5, 1107-1134.

[8] G. Di Fazio. $L^{p}$ estimates for divergence form elliptic equations with discontinuous coefficients. Boll. Un. Mat. Ital. A 10 (1996), no. 2, 409-420.

[9] F. Duzaar and G. Mingione. Gradient estimates via linear and nonlinear potentials. J. Funct. Anal. 259 (2010), no. 11, 2961-2998.

[10] F. Duzaar and G. Mingione. Gradient estimates via non-linear potentials. Amer. J. Math. 133 (2011), no. 4, 1093-1149.

[11] L. Evans. A new proof of local $C^{1, \alpha}$ regularity for solutions of certain degenerate elliptic p.d.e. J. Differential Equations 45 (1982), no. 3, 356-373.

[12] N. Fujii. Weighted bounded mean oscillation and singular integrals. Math. Japon. 22 (1977/78), no. 5, 529-534.

[13] E. Giusti. Direct methods in the calculus of variations. World Scientific Publishing Co., Inc., River Edge, NJ, 2003.

[14] L. Grafakos. Modern Fourier Analysis. Graduate Text in Mathematics Springer, second edition, 2009.

[15] V. Guliyev, S. Muradova, M. Omarova and L. Softova. Gradient estimates for parabolic equations in generalized weighted Morrey spaces. Acta Math. Sin. (Engl. Ser.) 32 (2016), no. 8, 911-924.

[16] V. Guliyev, M. Omarova, M. Ragusa and A. Scapellato. Commutators and generalized local Morrey spaces. J. Math. Anal. Appl. 457 (2018), no. 2, 1388-1402.

[17] L. Hoang, T. Nguyen and T. Phan, Gradient estimates and global existence of smooth solutions to a cross-diffusion system. SIAM J. Math. Anal. 47 (2015), no. 3, 2122-2177.

[18] T. Iwaniec. Projections onto gradient fields and $L^{p}$-estimates for degenerated elliptic operators. Studia Math. 75 (1983), no. 3, 293-312.

[19] A. Kairema. Two-weight norm inequalities for potential type and maximal operators in a metric space. Publ. Mat. 57 (2013), no. 1, 3-56. 
[20] J. Kinnunen and S. Zhou. A local estimate for nonlinear equations with discontinuous coefficients. Comm. Partial Differential Equations 24 (1999), no. 11-12, 2043-2068.

[21] Y. Komori and S. Shirai. Weighted Morrey spaces and a singular integral operator. Math. Nachr. 282 (2009), no. 2, 219-231.

[22] O. Ladyzhenskaya and N. Uraltseva. Linear and quasilinear elliptic equations. Translated from the Russian by Scripta Technica, Inc. Translation editor: Leon Ehrenpreis Academic Press, New YorkLondon, 1968.

[23] J. Lewis. Regularity of the derivatives of solutions to certain degenerate elliptic equations. Indiana Univ. Math. J. 32 (1983), no. 6, 849-858.

[24] T. Mengesha and N.C. Phuc. Global estimates for quasilinear elliptic equations on Reifenberg flat domains. Arch. Ration. Mech. Anal. 203 (2011), 189-216.

[25] T. Mengesha and N.C. Phuc. Weighted and regularity estimates for nonlinear equations on Reifenberg flat domains. J. Differential Equations 250 (2011), no. 5, 2485-2507.

[26] B. Muckenhoupt. Weighted norm inequalities for the Hardy maximal function. Trans. Amer. Math. Soc. 165 (1972) 207-226.

[27] S. Nakamura. Generalized weighted Morrey spaces and classical operators. Math. Nachr. 289 (2016), no. $17-18,2235-2262$.

[28] C. Neugebauer. Inserting Ap-weights. Proc. Amer. Math. Soc. 87 (1983), no. 4, 644-648.

[29] T. Nguyen and T. Phan. Interior gradient estimates for quasilinear elliptic equations. Calc. Var. Partial Differential Equations 55 (2016), no. 3, Art. 59, 33 pp.

[30] C. Pérez. On sufficient conditions for the boundedness of the Hardy-Littlewood maximal operator between weighted Lp-spaces with different weights. Proc. London Math. Soc. (3) 71 (1995), no. 1, $135-157$.

[31] C. Pérez and E. Rela. A new quantitative two weight theorem for the Hardy-Littlewood maximal operator. Proc. Amer. Math. Soc. 143 (2015), no. 2, 641-655.

[32] T. Phan. Regularity estimates for BMO-weak solutions of quasilinear elliptic equations with inhomogeneous boundary conditions. NoDEA Nonlinear Differential Equations Appl. 25 (2018), no. 2, Art. $8,49 \mathrm{pp}$.

[33] M. Ragusa. Regularity of solutions of divergence form elliptic equations. Proc. Amer. Math. Soc. 128 (2000), no. 2, 533-540.

[34] E. Sawyer. A characterization of a two-weight norm inequality for maximal operators. Studia Math. 75 (1982), no. 1, 1-11. 
[35] P. Tolksdorf. Regularity for a more general class of quasilinear elliptic equations. J. Differential Equations 51 (1984), no. 1, 126-150.

[36] K. Uhlenbeck. Regularity for a class of non-linear elliptic systems. Acta Math. 138 (1977), no. 3-4, 219-240.

[37] N. Uralt́ceva. Degenerate quasilinear elliptic systems. (Russian) Zap. Naučn. Sem. Leningrad. Otdel. Mat. Inst. Steklov. (LOMI) 7 1968, 184-222.

[38] M. Wilson. Weighted inequalities for the dyadic square function without dyadic $A_{\infty}$. Duke Math. J. 55 (1987), no. 1, 19-50.

G. Di Fazio, Dipartimento di Matematica e Informatica, Università di Catania, Viale A. Doria 6, 95125 Catania, Italy.

E-mail: giuseppedifazio@unict.it

T. Nguyen, Department of Mathematics, The University of Akron, 302 Buchtel Common, Akron, OH 44325, USA.

E-mail: tnguyen@uakron.edu 